\title{
Connectomics of Human Electrophysiology
}

\author{
Sepideh Sadaghiani ${ }^{1,2}$, Matthew J Brookes ${ }^{3}$ and Sylvain Baillet ${ }^{4}$ \\ 1. Department of Psychology, University of Illinois, Urbana-Champaign, IL, USA \\ 2. Beckman Institute for Advanced Science and Technology, University of Illinois, Urbana-Champaign, IL, \\ USA. \\ 3. Sir Peter Mansfield Imaging Centre, School of Physics and Astronomy, University of Nottingham, \\ University Park, Nottingham, NG72RD, UK. \\ 4. McConnell Brain Imaging Centre, Montreal Neurological Institute, McGill University, Montreal, QC, \\ Canada.
}

\begin{abstract}
We present both a scientific overview and conceptual positions concerning the challenges and assets of electrophysiological measurements in the search for the nature and functions of the human connectome. We discuss how the field has been inspired by findings and approaches from functional magnetic resonance imaging (fMRI) and informed by a small number of significant multimodal empirical studies, which show that the canonical networks that are commonplace in $\mathrm{fMRI}$ are in fact rooted in electrophysiological processes. This review is also an opportunity to produce a brief, up-to-date critical survey of current data modalities and analytical methods available for deriving both static and dynamic connectomes from electrophysiology. We review hurdles that challenge the significance and impact of current electrophysiology connectome research. We then encourage the field to take a leap of faith and embrace the wealth of electrophysiological signals, despite their apparent, disconcerting complexity. Our position is that electrophysiology connectomics is poised to inform testable mechanistic models of information integration in hierarchical brain networks, constructed from observable oscillatory and aperiodic signal components and their polyrhythmic interactions.
\end{abstract}

\section{1) Introduction}

Brain connectomics is a young and fast-growing sub-discipline of neuroscience that has transformed human brain mapping (Raichle, 2009; Sporns, 2010). More specifically, connectomics examines the macroscopic scale of functional and structural connectivity between cell assemblies. Spawned from MRI at the turn of the $21^{\text {st }}$ century and boosted by mathematical instruments such as graph theory, connectome studies aim to provide an integrative view of brain structure and function, in health and disease. Access to larger and deeper multimodal research data repositories and the growing sophistication of analytical methods have contributed to a fastevolving landscape of MRI-based derivations and interpretations of the human connectome (see e.g., Glasser et al., 2016). In this context, we interrogate the singular role and assets of electrophysiology in connectome research.

Here we define electrophysiological functional connectivity as a set of metrics that describe "high-level coupling across low-level networks". A low-level network is a circuit of interconnected cells spreading over $1 \mathrm{~cm}^{2}$ of cortex or more - a spatial scale that varies across 
the brain with local cell density, depth of the region and the main orientation of the current flow within that region (Murakami and Okada, 2006; Nunez and Srinivasan, 2006). Cell synchronization within such circuits increases local signal-to-noise ratio, which permits the extraction of the corresponding source signals with electrophysiological techniques. Because such local circuits are functionally relatively homogenous, we refer to them as "brain regions". High-level coupling constitutes signal interactions between these local circuits, across the brain. These interacting circuits, which are separated by a distance substantially greater than the spatial extent of each circuit in the network (i.e., typically at least $1 \mathrm{~cm}$ ), form the high-level networks at the core of the present review.

Electrophysiological techniques are historically the first to measure brain activity and remain the most accessible and most published techniques in basic (in both human and animal studies) and clinical neuroscience (Baillet, 2017). They consist of a remarkably diverse portfolio of methods that has evolved with decades of progress in sensing and computing technologies. Their specific strengths are 1) the ability to assess neural activity directly, contrasting indirect metabolic signals, 2) their millisecond temporal resolution (a consequence of the direct measurement), 3) their versatility to record at multiple spatial scales, from single cells to the whole brain, 4) the possibility to establish causal effects via concurrent neurostimulation, and 5) the growing availability, cost-effectiveness and data quality of portable, ambulatory instruments.

We also emphasize that electrophysiological data can be effectively combined with other types of neuroimaging data and technologies: from the relatively straightforward registration of electrophysiological recordings with structural MRI, to simultaneous scalp data collection with functional magnetic resonance (fMRI) or positron emission tomography (PET), all potentially synchronized with neurostimulation either intracranially or non-invasively (see e.g., (Driver et al., 2009) for a review).

This versatility and flexibility extend beyond human neuroscience. Electrophysiological techniques are available in vitro, in cell cultures, tissue slices and organoids (Trujillo et al., 2019). They also enable unique metrics in behaving animal models, simultaneously combined with calcium imaging or specific pharmacological and optogenetic manipulations (Kim et al., 2017). The ability to marry these ex-vivo or animal studies with, for example, whole-head non-invasive investigations in humans with electroencephalography (EEG) or magnetoencephalography (MEG) offers the powerful prospect to develop an understanding that spans from in-vitro models to human neuroimaging and behaviour, as a mechanistic common denominator across species and spatial and behavioural scales.

In sum, electrophysiology encompasses a set of cross-cutting scientific technologies and methods that provide unique access to the neural dynamics of brain systems. In principle, they can deliver unique insight on how functional communication is implemented biologically in brain networks, to enable complex behaviour over a broad range of time scales. Our goal here is to explain why these unique assets make electrophysiological approaches particularly pertinent to connectome research. 
Electrophysiological connectome studies are also uniquely positioned to address a wide range of clinical neuroscience questions. For instance, scalp and intracranial EEG are widely used in the epilepsy clinic (Henry, 2006). So far, the purpose has been to identify the origin of seizures, with the underlying hypothesis that a circumscribed onset zone would entrain the rest of the brain to generate a seizure. Recent epilepsy research has gradually evolved from a region detection approach towards a more network-oriented view, whereby in non-localizing cases, seizures may be the consequences of pathological interactions in an anatomically distributed seizure onset network (McCormick and Contreras, 2001). Electrophysiological investigations at multiple spatial scales in behaving patients (e.g., Despouy et al., 2020), combined with electrical stimulation at specific brain locations (Kim, 2016), are uniquely poised to reconcile complex brain network activity with patient behaviour in neurocognitive tasks (Thiery et al., 2020), and the realtime unfolding of seizure semiology (Moreau et al., 2020).

In neurodegenerative brain disease research, electrophysiological connectomics have the capacity to enable the early detection of pathophysiological processes affecting brain networks, before clinical symptoms emerge and before structural alterations are visible in MRI, at a lower cost than PET and with the anatomical specificity that emerging blood and other fluid tests lack (Jeromin and Bowser, 2017). In Alzheimer's disease for instance, very early alterations of synaptic regulation in cell assemblies may be detectable with electrophysiology before they provoke the larger-scale accumulation of beta-Amyloid plaques and Tau neurofibrillary tangles (Palop et al., 2006).

Similarly, in neuropsychiatry, electrophysiological connectomes have shown promise in the identification of putative markers of disease progression, and have potential to enable new approaches to the stratification of patients (Uhlhaas and Singer, 2010).

Taken together, electrophysiological techniques stand out with the unique potential to clarify the biological nature of network interactions across the widest possible range of temporal scales across the whole brain, from millisecond to days-long explorations. These techniques provide information in tissue cultures, in behaving animal models, in humans through development and aging, in premature new-borns and complex behaving adults, and from minimal, altered to full states of consciousness (e.g., Engemann et al., 2018).

Considering such unique assets, the exciting promise for basic and clinical neuroscience, and a long-standing body of trailblazing literature (Kujala et al., 2008; Schnitzler and Gross, 2005), one needs to question why electrophysiological studies to date have not been more pervasive and impactful in the general connectome literature. We review this question in depth, highlighting striking contributions of electrophysiology in connectome research, and suggesting pathways to mitigate the complexity and practical pitfalls of electrophysiological techniques. We emphasize the need for more electrophysiological connectome studies to advance knowledge of the biological mechanisms of information routing and integration by brain networks. Electrophysiology's direct sensitivity to neural activity enables the development, testing and 
1 validation of meaningful mechanistic models, which can inform study designs and inspire 2 innovative analytical methods.

To that end, we organized this position paper in 4 sections. In Section 2, we briefly outline 5 some of the methods that are available for electrophysiological data collection and the analysis 6 techniques commonly used in connectome investigations. We then discuss recent findings 7 relating to dynamic (time-resolved) functional connectivity derived from electrophysiological data, 8 reviewing current and emerging methods as well as nascent findings. In Section 3, we describe 9 why the rich and complex features of ongoing electrophysiological activity (i.e., not averaged with 10 respect to a stimulus input or behavioural event), although traditionally ignored, are meaningful, 11 both in terms of their relation to the structural and functional MRI connectome, and their 12 behavioural significance. We call for a leap of faith from connectome researchers to embrace this 13 richness in the electrophysiological signals available to them. After highlighting the main technical 14 challenges of connectivity measurements from time-resolved data, Section 4 proposes a 15 conceptual framework as an example to inspire and advocate for testable concepts and 16 mechanistic approaches to connectome studies with electrophysiology. 


\section{2) Measuring the electrophysiological connectome}

\section{1) Data modalities in human electrophysiology}

Measurements of electrophysiological high-level connectivity between low-level circuits (or brain regions) must necessarily provide 1) high fidelity, i.e. sufficient signal-to-noise ratio (SNR) to enable accurate characterisation of statistical interdependencies between signals from separate brain regions; and 2) sufficient spatial resolution to ensure that connectivity estimation between regions is not degraded substantially by spurious signal interactions ("leakage" or "crosstalk") from one region to another. With this in mind, electrophysiological measurements can be divided into two categories:

Non-invasive methods encompass electroencephalography (EEG) (Berger, 1929) and magnetoencephalography (MEG) (Cohen, 1972); the former measures electrical potential differences at the scalp surface generated by fluctuating current flow in the brain; the latter measures the corresponding magnetic induction generated by the same current fluctuations. Both probe electrophysiological activity in relatively large $(>50,000)$ populations of neurons (Murakami and Okada, 2006). The main neurophysiological generators are currents induced by post-synaptic potentials (local field potentials, LFPs) primarily from cortical pyramidal cells (Baillet, 2017). Mathematical modelling of scalp-level fields/potentials enables localisation of electrophysiological signals, and consequently inference on the activity within low-level (local) networks.

Invasive measurements, often collectively termed intracranial-EEG (iEEG), range from electrocorticography (ECoG) (where electrode arrays are placed under the dura mater on the brain surface) to depth electrodes targeting deeper structures. Measurement of localised electrical potential at each electrode site, relative to some reference, provides direct assessment of local electrical activity within the low-level circuit. Reference placement generally determines the size of the neuronal population from which the measurement is made. Measurements commonly reflect LFPs from cortical pyramidal cells (similar to MEG/EEG) summed over thousands of neurons (Rasch et al., 2009). However, some depth electrodes can also measure action potentials, including in behaving humans (Fried et al., 1997).

There is no single "best technique" to assess electrophysiological connectivity. Indeed, the technique of choice depends on the scientific question. Intracranial EEG typically provides the highest SNR, with high spatial resolution, but has limited spatial coverage of the brain volume. iEEG is highly invasive and therefore restricted to data from surgical patients who volunteer to participate in research. The implantation montage is idiosyncratic of each patient case, which challenges group studies and replication of findings. Further, the data collected may be affected by abnormal neural processes, since electrode locations are decided based on pathophysiological considerations. This issue can be remedied to some degree by excluding electrodes that are close to epileptic foci or have extensive epileptiform activity, as well as removing epochs with epileptiform activity from all electrodes. Crucially for connectome studies, spatial coverage of recordings is sparse in single individuals, which precludes whole-brain connectivity studies, although pooling across several patients can alleviate the issue.

The signals measured with MEG and EEG mirror those of iEEG, with the substantial benefits of providing synoptic coverage of the brain, noninvasively and both in patients and 
healthy participants. However, with extracranial sensors further away from brain sources, MEG and EEG have lower SNR compared to iEEG (especially at high frequency). Importantly for connectome derivations, multiple sensors pick up activity from the same electrical source in the brain - an effect known as volume conduction (in EEG) or field spread (in MEG) that introduces leakage between scalp measurement points. Inverse modelling (reconstructing source space estimates of brain current based on scalp-level field/potential measurement) of EEG/MEG generators improves this issue (Schoffelen and Gross, 2009). Nevertheless, the ill-posed nature of the inverse problem means that even in source space, leakage can still occur between signals generated by low-level networks of different regions, which complicates connectivity measurements (see also Section 2.2 and Table 2). Compared to EEG, MEG offers higher spatial accuracy since it is less sensitive to the geometry and conductance of head tissues. MEG is also less susceptible to biological artifacts (Baillet, 2017; Boto et al., 2019). However, MEG is also more expensive to purchase and operate, and is therefore less accessible. Significant progress on sensing technology promises the delivery of new, more flexible and affordable MEG instruments which have recently proved effective for connectivity measurement (Boto et al., 2021).

In Table 1, we summarise the similarities and differences within the family of electrophysiological recording techniques. Most importantly, all of these techniques can offer useful and high-fidelity measures of connectivity, which complement the structural and functional connectomes measured using MRI/fMRI.

22

\begin{tabular}{|c|c|c|c|c|c|c|}
\hline Modality & Mechanism & Coverage & $\begin{array}{l}\text { Temporal } \\
\text { resolution (TR) }\end{array}$ & Spatial resolution (SR) & Data quality & Practicality \\
\hline 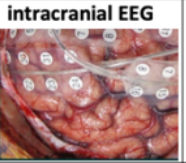 & $\begin{array}{l}\text { Electrodes placed on or } \\
\text { beneath the surface of } \\
\text { the brain to measure } \\
\text { localised electrical } \\
\text { potential. }\end{array}$ & $\begin{array}{l}\text { Electrodes placed } \\
\text { in a patch over a } \\
\text { specific area of } \\
\text { cortex. Coverage } \\
\text { therefore relatively } \\
\text { limited. }\end{array}$ & $\begin{array}{l}\text { TR is } 1 \mathrm{~ms} \text { or } \\
\text { better. }\end{array}$ & $\begin{array}{l}\text { For shallow sources SR is limited } \\
\text { by separation of electrodes. For } \\
\text { deeper sources, volume } \\
\text { conduction means a single source } \\
\text { can be detected at multiple } \\
\text { electrodes so modelling required. }\end{array}$ & $\begin{array}{l}\text { The gold standard for } \\
\text { data quality. Because } \\
\text { electrodes are placed } \\
\text { directly in the brain, we } \\
\text { achieve extremely high } \\
\text { SNR. }\end{array}$ & $\begin{array}{l}\text { Requires invasive } \\
\text { surgery to place } \\
\text { electrodes. Limited to } \\
\text { clinical cases (e.g. } \\
\text { epilepsy) or } \\
\text { experimental animals }\end{array}$ \\
\hline EEG & $\begin{array}{l}\text { Electrodes placed in } \\
\text { conductive contact } \\
\text { with the scalp and } \\
\text { potential differences } \\
\text { between each } \\
\text { electrode and a } \\
\text { reference are } \\
\text { measured. }\end{array}$ & $\begin{array}{l}\text { Whole brain } \\
\text { coverage possible } \\
\text { with even cortical } \\
\text { sensitivity. } \\
\text { Sensitivity declines } \\
\text { with depth into the } \\
\text { head. }\end{array}$ & $\begin{array}{l}\text { TR is } 1 \mathrm{~ms} \text { or } \\
\text { better. }\end{array}$ & $\begin{array}{l}\text { The skull has low electrical } \\
\text { conductivity, meaning patterns of } \\
\text { electrical potential at the scalp } \\
\text { are "smeared" out. This is hard to } \\
\text { model, and SR thus depends on } \\
\text { complex head models. Spatial } \\
\text { information is often distorted and } \\
\text { SR is very limited. }\end{array}$ & $\begin{array}{l}\text { Signal amplitude is } \\
\text { reduced by the skull so } \\
\text { SNR lower than ECoG. } \\
\text { EEG is also sensitive to } \\
\text { biological artifact e.g. } \\
\text { electrical signal in } \\
\text { muscles; this degrades } \\
\text { connectivity estimation. }\end{array}$ & $\begin{array}{l}\text { Electrical contact with } \\
\text { scalp requires } \\
\text { significant set up time. } \\
\text { Subjects can move } \\
\text { freely during a scan } \\
\text { (albeit at the cost of } \\
\text { SNR). Systems adapt } \\
\text { to any head size. }\end{array}$ \\
\hline MEG & $\begin{array}{l}\text { Magnetic field } \\
\text { detectors } \\
\text { (superconducting } \\
\text { quantum interference } \\
\text { devices) placed close } \\
\text { to the scalp to } \\
\text { measure the magnetic } \\
\text { fields generated by } \\
\text { cortical current flow }\end{array}$ & $\begin{array}{l}\text { Whole brain } \\
\text { coverage possible } \\
\text { but coverage can } \\
\text { be inhomogeneous } \\
\text { (due to different } \\
\text { head shapes in a } \\
\text { "one size fits all" } \\
\text { scanner). Sensitivity } \\
\text { declines with depth }\end{array}$ & $\begin{array}{l}\text { TR is } 1 \mathrm{~ms} \text { or } \\
\text { better. }\end{array}$ & $\begin{array}{l}\text { Magnetic fields pass through the } \\
\text { skull relatively undistorted; } \\
\text { modelling is therefore simple and } \\
\text { MEG has better SR than EEG. SR } \\
\text { limited mainly by proximity of } \\
\text { sensors to the scalp; the need for } \\
\text { thermal insulation of sensors } \\
\text { means sensors distal }(\sim 2 \mathrm{~cm}) \text { and } \\
\text { this makes field patterns diffuse. }\end{array}$ & $\begin{array}{l}\text { Affected by brain to } \\
\text { sensor distance; high } \\
\text { SNR in adults but } \\
\text { reduced in subjects with } \\
\text { small heads. } \\
\text { Lower sensitivity to } \\
\text { biological artifact than } \\
\text { EEG but SNR reduced by } \\
\text { subject movement. }\end{array}$ & $\begin{array}{l}\text { Simple to set up and } \\
\text { run. However, } \\
\text { subjects have to } \\
\text { remain still for long } \\
\text { periods. Systems are } \\
\text { expensive. Lifetime } \\
\text { compliance difficult } \\
\text { due to "one-size-fits- } \\
\text { all" system. }\end{array}$ \\
\hline & $\begin{array}{l}\text { Equivalent to MEG but } \\
\text { employs lightweight } \\
\text { "on-scalp" (non- } \\
\text { superconducting) } \\
\text { sensors which get } \\
\text { closer to the scalp } \\
\text { surface }\end{array}$ & $\begin{array}{l}\text { Whole brain } \\
\text { coverage possible. } \\
\text { Scalp mounted } \\
\text { sensors means } \\
\text { even coverage. } \\
\text { Sensitivity declines } \\
\text { with depth. }\end{array}$ & $\begin{array}{l}\text { Limited by } \\
\text { sampling speed; } \\
\text { most commonly } \\
\text { used sensor } \\
\text { provides TR of } \\
5 \text { ms.) }\end{array}$ & $\begin{array}{l}\text { Maintains advantages of simple } \\
\text { modelling, but because sensors } \\
\text { get closer to the head, the field } \\
\text { patterns are less diffuse. This } \\
\text { means fundamentally higher SR } \\
\text { compared to conventional MEG } \\
\text { or EEG. }\end{array}$ & $\begin{array}{l}\text { Higher SNR than both } \\
\text { MEG and EEG because } \\
\text { sensors get close to the } \\
\text { head (but not as high as } \\
\text { ECoG). Retains } \\
\text { advantages with respect } \\
\text { to biological artifacts. }\end{array}$ & $\begin{array}{l}\text { Simple to set up and } \\
\text { run. Subjects can } \\
\text { move freely. Systems } \\
\text { adapt to any head } \\
\text { shape. However, } \\
\text { nascent technology } \\
\text { not widely available. }\end{array}$ \\
\hline fMRI & $\begin{array}{l}\text { Measures the blood } \\
\text { oxygenation level } \\
\text { dependent response to } \\
\text { localised changes in } \\
\text { metabolism }\end{array}$ & $\begin{array}{l}\text { Excellent coverage } \\
\text { of the entire brain, } \\
\text { including deep } \\
\text { structures. }\end{array}$ & $\begin{array}{l}\text { TR limited to } 5 \\
S \text { by the latency } \\
\text { and longevity of } \\
\text { the } \\
\text { haemodynamic } \\
\text { response. }\end{array}$ & $\begin{array}{l}\text { Excellent spatial resolution (e.g. } 1 \\
\text { mm) since the haemodynamic } \\
\text { response is tightly spatially } \\
\text { coupled to cellular activity. } \\
\text { However, images acquired } \\
\text { quickly can be spatially distorted. }\end{array}$ & $\begin{array}{l}\text { Depends on field } \\
\text { strength. Both SNR and } \\
\text { the relative sensitivity to } \\
\text { capillaries (rather than } \\
\text { large veins) improve } \\
\text { with higher field. }\end{array}$ & $\begin{array}{l}\text { Simple to set up and } \\
\text { run. Subjects have to } \\
\text { remain still for long } \\
\text { periods. Acoustic noise } \\
\text { can limit paradigms } \\
\text { possible }\end{array}$ \\
\hline
\end{tabular}

Table 1 -- Methods to measure electrophysiological activity 


\section{2) Current approaches to electrophysiological connectivity assessment}

Regardless of how they are measured, electrophysiological signals are dominated by "oscillations" which exist across a broad $(0-1000 \mathrm{~Hz})$ frequency range. Most signal power is concentrated in the low frequencies $(1-100 \mathrm{~Hz})$, and this range is subdivided into bands, traditionally defined as delta $(1-4 \mathrm{~Hz})$, theta $(4-8 \mathrm{~Hz})$, alpha $(8-13 \mathrm{~Hz})$ beta $(13-30 \mathrm{~Hz})$ and gamma $(30-100 \mathrm{~Hz})$. At very low frequencies, infra-slow fluctuations can also be measured. This rich multi-spectral signal offers myriad metrics by which to compute statistical interdependencies between signals derived from separate brain locations. Such metrics, because they are applied directly to electrophysiological data, offer perhaps the best means to understand the mechanisms of functional coupling. However, equally they also mean that the electrophysiological connectivity literature is complex and often dominated by high-level methodological discussions which can obfuscate the neuroscientific findings. To make this literature more accessible, we provide a (nonexhaustive) introduction to the available connectivity measures and refer the reader to their indepth discussion in dedicated literature (Bastos and Schoffelen, 2016; Cao et al., 2021; Jensen and Colgin, 2007).

Broadly, electrophysiological connectivity metrics can be split into two categories, withinfrequency band (e.g., alpha-to-alpha (cf. Bastos and Schoffelen, 2016)) and between frequency bands (e.g., alpha-to-gamma (cf. Jensen and Colgin, 2007)). Two classes of within-frequency connectivity metric are currently popular: fixed phase relationships and amplitude correlation measures. These disparate techniques are thought to provide insight into two separate modes of functional connectivity ((Engel et al., 2013; Helfrich et al., 2016; Mostame and Sadaghiani, 2020), but note that some phase coupling measures, most notably coherence, may also be sensitive to correlated fluctuations in amplitude between nodes). For between-frequency measures, there are three typical families of techniques: phase-phase, amplitude-amplitude, and phase amplitude coupling, the latter being the most commonly applied. We summarise these measures in Table 2, with a quick, practical overview of their respective physical foundations, alongside the primary methodological considerations that must be taken into account prior to using them. 


\begin{tabular}{|c|c|c|c|c|}
\hline Metric class & Measurement & Mechanism & Considerations & Methods of application \\
\hline \multirow[t]{2}{*}{$\begin{array}{l}\text { Within } \\
\text { frequency } \\
\text { band }\end{array}$} & & $\begin{array}{l}\text { We seek a fixed phase relationship between } \\
\text { band limited signals, from spatially } \\
\text { separated brain regions. }\end{array}$ & $\begin{array}{l}\text { Should always be applied in source space to } \\
\text { mitigate problems with volume conduction } \\
\text { (EEG) or field spread (MEG) } \\
\text { Signal 'leakage' between regions can lead to } \\
\text { artificially high phase coupling metrics. } \\
\text { Consequently leakage correction is required. }\end{array}$ & $\begin{array}{l}\text { Coherence; phase locking value; phase } \\
\text { difference derivative - all assess fixed phase } \\
\text { relationships but are affected by signal } \\
\text { leakage) } \\
\text { Imaginary Coherence; phase lag index - } \\
\text { inherently correct for leakage by removing } \\
\text { zero phase lag interactions. }\end{array}$ \\
\hline & DWMPlitude coupling & $\begin{array}{l}\text { The amplitude (or power) envelope of band } \\
\text { limited oscillations is computed and we seek } \\
\text { to find correlations in the amplitude } \\
\text { envelope between regions }\end{array}$ & $\begin{array}{l}\text { Should always be applied in source space. As } \\
\text { with phase interactions, 'leakage' between } \\
\text { regions can lead to artificially high correlation } \\
\text { and so leakage correction is required. }\end{array}$ & $\begin{array}{l}\text { Amplitude coupling is typically measured via } \\
\text { Pearson correlation between envelope signals. } \\
\text { Prior to this, to mitigate source leakage, signal } \\
\text { orthogonalisation should be applied using } \\
\text { either pairwise or symmetric methods. }\end{array}$ \\
\hline \multirow[t]{3}{*}{$\begin{array}{l}\text { Between } \\
\text { Frequency } \\
\text { bands }\end{array}$} & & $\begin{array}{l}\text { In general a relationship is sought whereby } \\
n \text { rhythmic cycles at one location temporally } \\
\text { coincide with } m \text { rhythmic cycles at a second }\end{array}$ & $\begin{array}{l}\text { Commonly used in mathematical models of } \\
\text { neural oscillations but rarely applied to real } \\
\text { data. }\end{array}$ & $\begin{array}{l}\text { Measure directly. } \\
\text { However care must be taken to mitigate } \\
\text { spurious effects due to leakage. }\end{array}$ \\
\hline & 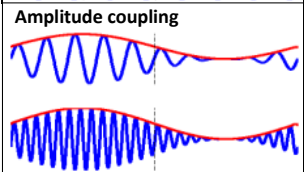 & $\begin{array}{l}\text { Equivalent to within band amplitude } \\
\text { coupling; one seeks correlation between the } \\
\text { amplitude envelope of frequency A at } \\
\text { location one, and the amplitude envelope } \\
\text { of frequency B at location two }\end{array}$ & $\begin{array}{l}\text { Should always be applied in source space. } \\
\text { Can be affected by leakage but only in the } \\
\text { case of true cross frequency correlation; e.g. if } \\
\text { true correlation exists at region A, and signals } \\
\text { leak from A to B, one will infer spurious cross } \\
\text { frequency effects between A and B. }\end{array}$ & $\begin{array}{l}\text { Measure by simply Pearson correlation. } \\
\text { However care must be taken to mitigate } \\
\text { spurious effects due to leakage. }\end{array}$ \\
\hline & 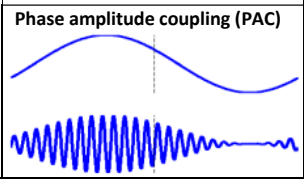 & $\begin{array}{l}\text { The phase of a low frequency oscillation is } \\
\text { coupled to the amplitude of a higher } \\
\text { frequency oscillation. This can occur within } \\
\text { a single brain region, or across regions (e.g. } \\
\text { low frequency phase in region A driving high } \\
\text { frequency amplitude in region B. }\end{array}$ & $\begin{array}{l}\text { Should always be applied in source space. } \\
\text { Can be affected by leakage if true PAC exists; } \\
\text { e.g. if genuine PAC exists at region A, and } \\
\text { signals leak from A to B, one infers spurious } \\
\text { PAC between A and B. }\end{array}$ & $\begin{array}{l}\text { Measure by simply Pearson correlation. } \\
\text { However care must be taken to mitigate } \\
\text { spurious effects due to leakage. } \\
\text { Care must be taken to avoid spurious effects } \\
\text { in the case of non-sinusoidal oscillations } \\
\text { which, following Fourier analysis, can manifest } \\
\text { as within region PAC. }\end{array}$ \\
\hline
\end{tabular}

Table 2 -- Mathematical techniques to characterize connectivity between electrical signals derived from separate brain regions.

Importantly, and as with all types of biosignal analysis, the biggest caveat to electrophysiological connectivity stems from the inherent limits to data quality, most notably as they pertain to spatial resolution and signal leakage - especially for MEG/EEG. Reliable and robust methods to mitigate leakage have been introduced in recent years. Most of these rely on the idea that leakage necessarily manifests as a relationship between signals with zero time lag (i.e. a linear correlation between measured oscillations, or alternatively coherence with zerophase lag). When probing for fixed phase relations between signals, such zero-lag effects can be easily removed - for example by taking only the imaginary part of a coherence calculation (Nolte et al., 2004), or via use of the phase lag index (Stam et al., 2007). Equivalently, one can also control leakage via the mathematical process of "orthogonalization" (Colclough et al., 2015; Hipp et al., 2012) Many methods to do this exist, the simplest involving a simple regression; however arguably the most elegant is the symmetric orthogonalisation introduced by Colclough et al, in which linear relations between all possible pairs of regions (e.g. in a parcellation) are eliminated. Note that a more complete technical comparison of these approaches can be found in O'Neill et al (2017a). However, as discussed in Section 4, we emphasize and acknowledge that whilst this pragmatic approach does reduce leakage, genuine zero-phase lag delays are commonly observed between neurophysiological signals, even between distant brain nodes, and these true interactions are lost using these techniques. Hence, interpretation of data following removal of zero phase-lag effects needs to take such blind-spot effect into consideration. A second methodological caveat is signal artifacts affecting data quality, such as from muscle activity or movements. These artifacts are typically worse in EEG, although still significant in MEG (especially in terms of head movements in the MEG sensor array), while essentially minimal in 
iEEG. Implementations of these techniques to suppress leakage are available in free and commercial software to mitigate these unwanted signals.

Finally, we note that in addition to the class of metrics defined above, which estimate connectivity without indicating the "direction" of information flow, a number of directed measures have been developed (Bastos and Schoffelen, 2016; Cao et al., 2021). These include but are not limited to Granger causality, partial directed coherence and dynamic causal modelling. Such measures exploit the high temporal resolution of electrophysiological measurements to infer the average (over some time window) direction of information flow between two regions. These techniques are typically more complex and difficult to deploy in practice, especially over longer data length, essentially because of sophisticated parameter identification requirements, but nevertheless offer a means to assess directionality in brain networks in relation to cognition and behaviour. In the future these directed techniques should be applied in the field of whole-brain connectomics.

A schematic overview of the basic methodology to process electrophysiological data to derive an electrophysiological connectome measure is shown in Figure 1.

Data processing pipeline for connectome modelling in electrophysiological data

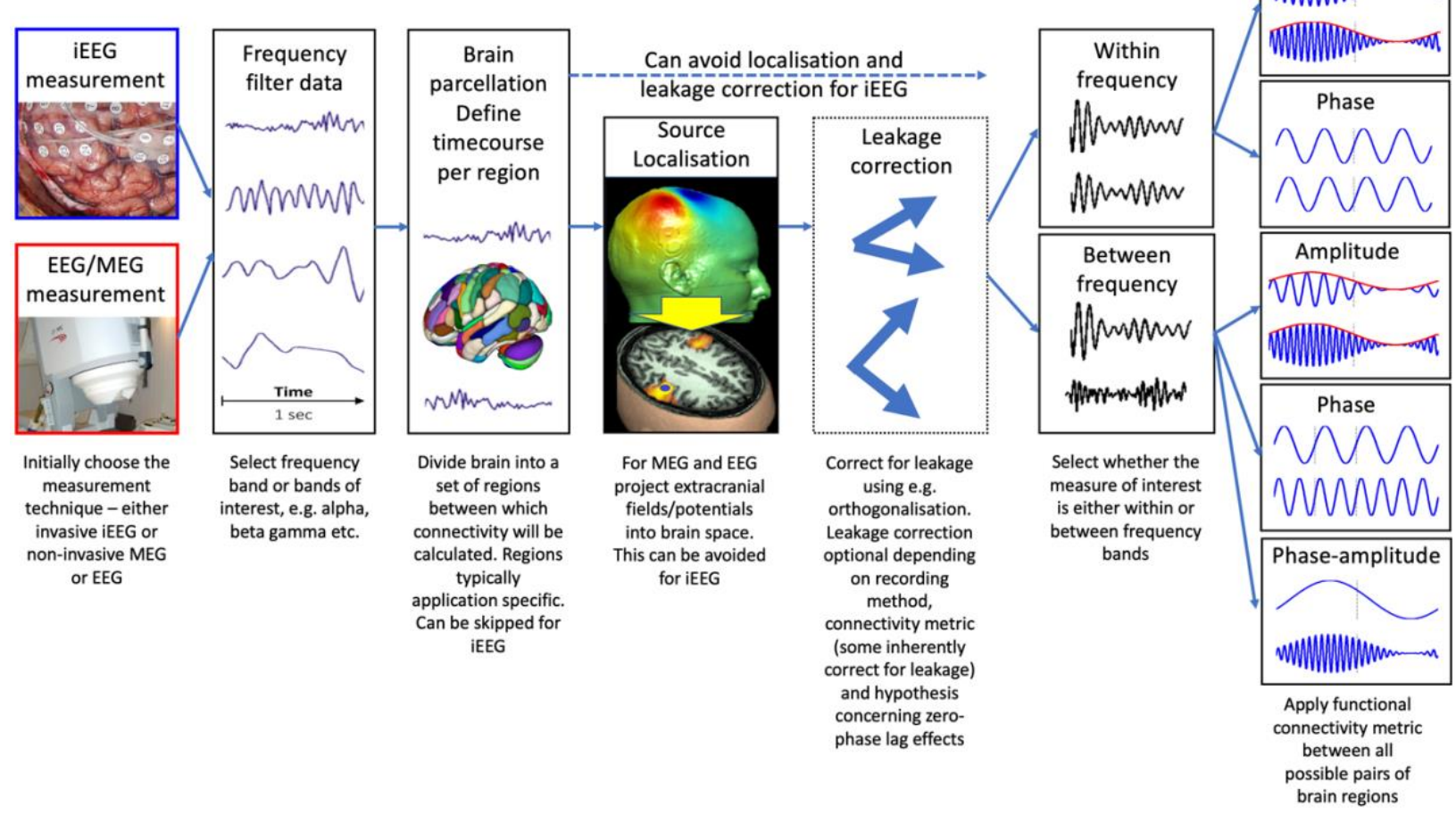

Figure 1 -- a schematic overview showing the process by which connectivity is calculated in 


\section{3) Towards a dynamic, time-resolved connectome}

In the context of large-scale connectomics, the connectivity metrics described above are

\section{Sliding window approaches}

At the simplest level, dynamic connectivity can be calculated via a 'sliding' window; i.e. one calculates connectivity between regions using the methods described in Table 2, but applied to a small segment (typically a few seconds) of data. This window of interest is then moved in time to track dynamics of that connection. This approach developed in fMRI (Chang and Glover, 2010) has been used extensively in electrophysiology (O'Neill et al., 2017a; cf. Section 3.3). A key point is that the utility afforded by such a technique depends on the length of the window; this, in turn, depends on the extent to which one can get a reliable metric of connectivity in a short time frame, which itself depends on the number of degrees of freedom in the signal.

The number of temporal degrees of freedom, $n$, in a signal is estimated as $n=2 B_{w} D$, where $B_{w}$ is the signal bandwidth and $D$ is the width, in time, of the window. Whereas the bandwidth of a ('classically' acquired) fMRI signal is $\sim 0.25 \mathrm{~Hz}$ (Yuen et al., 2019) the nominal bandwidth of the electrophysiological signal is at least $100 \mathrm{~Hz}$ (and can be much larger with reproducible signals up to and including $1 \mathrm{KHz}$ ). This means that (at face value) time windows for sliding-window based connectivity measures can be $\sim 400$ times shorter in electrophysiology compared to fMRI. This, in turn, makes electrophysiology the technique of choice for dynamic functional connectivity measurements. In practice, the electrophysiological signal contains different features in different bands, and one often looks to compute connectivity within narrowband signals (e.g., alpha, beta, gamma bands). This means that the improvement in time resolution over $\mathrm{fMRI}$ is not as dramatic. Nevertheless, even for the narrowest bands (e.g., the 8$13 \mathrm{~Hz}$ alpha band) the bandwidth remains at $5 \mathrm{~Hz}$, affording at least a 20-fold improvement in temporal resolution over fMRI.

However, despite the high bandwidth of electrophysiological signals, the width of the window for sliding window approaches remains a fundamental limitation; one really wants the window width to match the timescale of network fluctuations in the brain. However, in practice this is almost certainly unknown, and may change over the course of an experiment. Likewise, it may be different for different networks and vary with the age, or pathological state of the participant. It is also possible that the timescale of fluctuations in connectivity may simply be too short for viable 
measures of connectivity within the window (e.g., for alpha band, a 1-s window, which is still a long time compared with cognitive processing, only contains 10 degrees of freedom). For these reasons, whilst the sliding window remains a useful and conceptually straightforward tool, it is likely that other methods could better exploit the high temporal resolution that electrophysiology affords.

\section{Beyond the sliding window}

A number of techniques have attempted to examine connectivity "moment-to-moment", i.e., gain an estimate of functional connectivity for every sample in an electrophysiological time course. Here, an available technique is the phase-difference-derivative (PDD) (Breakspear et al., 2004). Briefly, PDD probes the existence of a fixed phase relationship; the instantaneous phase of signals from distal regions is acquired and the difference between them measured over time. The derivative of this phase difference indicates whether the phase difference is changing. If the difference derivative is zero, a transient fixed phase relation is implied. In unaveraged or resting state task free data, PDD and similar metrics tend to be unstable, and one ends up averaging over time windows which ultimately, leads to the same problem faced by sliding windows. However, in task-based studies, assuming the same experimental paradigm is repeated many times, PDD can be averaged over trials, an approach that has offered useful insights. For example, Tewarie et al. (2019) showed that high amplitude beta oscillations in the motor cortex were related to increased levels of connectivity between the primary motor cortex and other brain regions within the sensorimotor system.

One technique, developed in recent years, which is able to sidestep the windowing problem - even in resting state data - is based on hidden Markov modelling (HMM; Baker et al., 2014; Woolrich et al., 2013) (to date this technique has been applied mostly to MEG, but recent papers have employed it for both EEG and fMRI (Hunyadi et al., 2018)). The HMM assumes that electrophysiological data are governed by a series of mutually exclusive hidden "states". This means that at any one point in time, the brain can be said to exist in a specific state. The state sequence is Markovian (meaning that the state modelled is dependent only on the state immediately preceding it). In its simplest form, the HMM might describe states in a single electrophysiological time course; each state could be described by a Gaussian distribution from which the electrophysiological data are extracted. If, at some time point $t$, the observed electrophysiological data are most likely drawn from the Gaussian describing State 1, then State 1 would be assigned to time point $t$. Conversely, if the Gaussian from State 2 is most likely, state 2 would be assigned, and so on. This model can be expanded to encompass multivariate data where one includes all electrophysiological time courses from regions across the whole brain (here each state is governed by a multivariate mean and covariance (Baker et al., 2014)). More recent versions of the model also enable time embedding (Vidaurre et al., 2018), enabling states to be governed by a complete spatial, temporal and spectral description of brain activity.

Using this unsupervised approach, the identification of when the brain enters or leaves a specific state is automated, and because states are entered more than once, one can average data across many visits to the same state - improving SNR. In this way, we sidestep the 'classical' problem of having to average within predefined and largely meaningless sliding windows, since 
the HMM automatically selects the time windows in a data-driven manner. As we discuss further below, the HMM is offering a new means to capture brain network dynamics at very rapid timescales. We also discuss in Section 4 a conceptual physiological framework that -as an example of such frameworks- motivates hypothesis-driven selection of connectivity measures and may inspire the development of future electrophysiological connectivity measures in a timeresolved manner.

\section{Considerations for statistical assessments of significance}

\section{3) Exploiting ongoing electrophysiological activity to define electrophysiological connectomes}

In this section, we argue that many common electrophysiological analyses, on their own, fall short of providing a comprehensive mechanistic understanding of brain-behaviour relationships. Electrophysiological measurements are typically repeated many times, and data is averaged across trials to detect possible effects relative to a "baseline" period of reference. In most electrophysiological studies, the baseline is discarded, treating ongoing brain dynamics as "noise". Here, we argue in favour of exploiting in full, rather than "correcting away", contributions of ongoing neural processes and their spatial organization to electrophysiological recordings. We use the term "ongoing" in a broad sense to refer to all neural processes (both during rest and tasks) not captured by stimulus (or response)-locked and trial-averaged approaches.

Several observations motivate a comprehensive approach that incorporates ongoing connectivity. Firstly, ongoing processes constitute the strongest portion of the power spectrum of electrophysiological activity. These processes are spatio-temporally well organized, although they have been historically considered as generated by unstructured noise. Secondly, ongoing 
processes are of functional significance for behaviour. Below, we first lay out why common electrophysiological analyses are often blind to these consequential properties of ongoing activity. In Subsections 3.1 and 3.2, we elaborate on the spatial organization of ongoing electrophysiological activity (i.e. whole-brain pattern of connection strength across region-pairs), and discuss its behavioural significance. In Subsection 3.3 we review briefly the latest evidence on the dynamics of ongoing activity.

\section{Common "corrections" of baseline}

In task-based electrophysiological, local activation levels are typically assessed as eventrelated potentials or fields (ERPs and ERFs) and/or event-related spectral perturbations (ERSPs) that are corrected relative to pre-stimulus baseline or expressed as contrasts across experimental conditions or groups of participants. Both average evoked responses and their contrasts are blind to brain activity present at prestimulus baseline. Further, and by design, ERP/ERFs and ERSPs capture consistency over trials and are insensitive to the variability of evoked responses that may result from ongoing activity. Similarly, when assessing electrophysiological connectivity, coupling measures such as PLV and coherence are commonly normalized to prestimulus timepoints and designed to quantify cross-trial consistency. Task-related connectivity changes are thus commonly studied in a way that treats ongoing electrophysiological processes as "noise". It is also important to note that the often-chosen approach to study connectivity across a small number of task-related sensors or sources of interest neglects the distributed spatial organization of electrophysiological data.

\section{1) Ongoing electrophysiological activity has an intrinsic spatio-temporal organization}

An important aspect that invites going beyond the above-described "corrections" of ongoing activity is that this activity is spatio-temporally organized rather than merely reflecting unstructured noise. Below, we review electrophysiological investigations that have established the presence of a stable spatial organization of connectivity, paralleling earlier observations from functional and structural MRI. Specifically, we discuss the presence of "canonical" Intrinsic Connectivity Networks (ICNs, each comprising a specific distributed set of strongly interconnected brain regions, as initially described in MRI studies). We also discuss connection-wise quantitative comparisons of electrophysiological and MRI-based connectivity, and the context-invariant intrinsic nature of electrophysiological connectomes. Further conceptual and mechanistic considerations are proposed in Section 4, to inspire more research on these questions that make electrophysiological connectomics stand out uniquely.

\section{Intrinsic spatial organization in intracranial electrophysiology}

To establish the presence of ICN organization independently of the potential impact of source leakage, we first consider human intracranial recordings. Although intracranial studies typically lack the whole-brain coverage required for a comprehensive depiction of ICNs, individual studies have confirmed the existence of specific ICNs depending on the available electrode placement. High intra-network connectivity during task-free resting state has been reported across regions of the somatomotor ICN (Hacker et al., 2017; He et al., 2008; Ko et al., 2013; 
Weaver et al., 2016), the visual and auditory ICNs (Ko et al., 2013; Nir et al., 2008), and higherorder ICNs including the default mode (DM), dorsal attention (DAT), and fronto-parietal executive control (FP) networks (Hacker et al., 2017; Kucyi et al., 2018). Beyond within-ICN connectivity, more comprehensive intracranial connectome characterization includes the anti-correlation across the DM network and "task-positive" areas (Hacker et al., 2017; Keller et al., 2013), as well as observation of whole-brain connectomes (full connectivity matrix) by pooling available electrode-pairs over many subjects (Betzel et al., 2019; Figure 2C). The latter study reports a significant albeit moderate association between the group-level fMRI connectome and the pooled whole-brain ECoG connectome in all canonical frequency bands. Of note, although many studies that seek similarity with fMRI-derived FC focus on slow co-fluctuations of high-gamma power, the above-listed studies extend to amplitude coupling of other oscillation frequencies as well as measures that involve oscillation phase. In summary, human iEEG studies provide confidence regarding the presence of an intrinsic spatial organization of ongoing electrophysiological connectivity across oscillation frequencies and connectivity measures, and also lend support to the spatial network organisation often reported in fMRI.

\section{Intrinsic spatial organization in MEG/EEG}

With this confidence in an intrinsic spatial organization established, we turn to MEG and EEG source space studies of whole-brain connectomes. Numerous MEG amplitude-coupling studies offer converging evidence for the presence of sensory/motor (SM, VIS, AUD) and higherorder (DM, DAT, VAT, FP) ICNs using seed-based correlations (de Pasquale et al., 2010; Hipp et al., 2012, p. 2012; Figure 2A), temporal ICA (Brookes et al., 2011; Fig 2B) and discrete coactivation states (Baker et al., 2014; Figure $3 A$ and Section 3.3). While some of these studies use broadband signals, those that focus on distinct frequency bands often report the $\alpha$ and/or $\beta$ bands to be most dominant in reflecting ICNs. Further, while amplitude coupling has been the more commonly used connectivity mode in MEG resting state connectome studies, MEG phase coupling shows a similar spatial distribution anchored by ICNs (see below for (Tewarie et al., 2016) comparing both modes to fMRI FC). We discuss in Section 4, how these seemingly disparate observations from amplitude- and phase-coupling may come together, and how derivatives from cross-frequency, phase-amplitude coupling measures likewise provide ICNs akin to $\mathrm{fMRI}$ (Florin and Baillet, 2015; Figure 4E). Finally, in spite of stronger susceptibility to volume conduction compared to MEG, EEG likewise robustly reflects the intrinsic spatial organization of the connectome (Wirsich et al., 2021), backed by cross-modal convergence with fMRI and dMRI as described below.

\section{Robust but moderate spatial similarity of static MEG/EEG- to MRI-based connectomes.}

Beyond qualitative description of electrophysiological ICNs and connectomes, the connection-wise correspondence to MRI-derived connectivity patterns can be quantified when using the same brain parcellation atlas across data modalities. Such quantitative descriptions are useful because they inform conclusions regarding neurobiological reasons for convergence/divergence of connectivity patterns across data modalities (we thus include some effect sizes below). Such comparative studies have been discussed extensively in a previous paper (Sadaghiani and Wirsich, 2019). Here, we highlight some key observations. 
Connection-wise spatial similarity of static source-space MEG/EEG connectomes to the fMRI-derived connectome has been firmly established in numerous studies but is modest in effect size. This cross-modal similarity is significant but small at the level of individual subjects (Hipp and Siegel, 2015; MEG amplitude coupling). Effect sizes are considerably larger when comparing group-average $\mathrm{MEG} / \mathrm{EEG}$ and $\mathrm{fMRI}$-derived connectomes; across concurrently recorded $\mathrm{EEG}$ and fMRI connectomes the spatial correspondence is reported at $r=0.3$ for most bands (Wirsich et al., 2017; EEG imaginary phase coupling; Figure 2D). These effect sizes are similar to those discussed above for group-level iEEG connectomes (Betzel et al., 2019; Fig. 2C). Importantly, cross-modal connectome association has been firmly reproduced with the same moderate effect size in concurrent EEG-fMRI in numerous settings, from 64 to 256 EEG scalp electrodes and from 1.5 to $7 \mathrm{~T} \mathrm{MRI} \mathrm{field} \mathrm{strengths} \mathrm{(Wirsich} \mathrm{et} \mathrm{al.,} \mathrm{2021;} \mathrm{Figure} \mathrm{2D).} \mathrm{Interestingly,} \mathrm{while} \mathrm{MEG} \mathrm{group-}$ average connectomes in specific frequency bands show moderate resemblance to fMRI connectivity ( $r$ up to 0.35 for amplitude coupling and up to 0.24 for phase coupling depending on frequency band), associations double in size when jointly considering linear and nonlinear combinations of frequency-specific MEG connectomes (Tewarie et al., 2016). Further, jointly considering amplitude- and phase-coupling predicts the fMRI connectome at $r=0.73$. Thus, different canonical frequency bands and electrophysiological coupling modes may be associated with unique components of FC in fMRI. It is possible, even likely, that fMRI represents a nonlinear combination of electrophysiological coupling phenomena, further highlighting the advantage of electrophysiological modalities in dissociating the contribution of such phenomena to the functional connectome.

The above-described increase in MEG/EEG to fMRI spatial similarity from individual to group-level connectomes suggests that this similarity is driven by robustly shared features, i.e., the consensus across the population. The most robust tracts of the structural connectome play a key role in such FC consensus as a shared backbone. Indeed, both amplitude-, phase- and phase-amplitude coupling in all canonical frequency bands are stronger between nodes with direct or indirect structural connections (derived from diffusion-weighted MRI, dMRI) than those without such connections, even after accounting for spatial proximity (Chu et al., 2015; EEG phase coupling and amplitude coupling; Florin and Baillet, 2015; MEG phase-amplitude coupling). A study of the alpha band confirmed a close structure-function relationship both for group-average and individual connectomes $(r=0.48)$ (Finger et al., 2016; EEG phase coupling). A substantially higher association was achieved in this study between observed EEG connectomes and simulated EEG connectomes modelled from dMRI connectivity $(r=0.74)$. Importantly, such modelling approaches also provide mechanistic insights into the contribution of structural to electrophysiological connectome organization (e.g. Cabral et al., 2014; cf. Ritter et al. in this Special Issue). Interestingly, when the structure-function relationship for fMRI and EEG is directly compared, electrophysiology may match dMRI-derived connectivity better than fMRI (Deligianni et al., 2016; Wirsich et al., 2017; concurrent EEG-fMRI). The correspondence to dMRI-derived connectivity gives additional methodological credibility to electrophysiological source-space connectomes and points to a substantial contribution of structural connectivity.

Importantly, while noise and confounds in both electrophysiology and fMRI certainly contribute to the often modest size of their cross-modal association, the connectome 
dissimilarities are likely biologically meaningful. The vastly different temporal characteristics of $\mathrm{fMRI}$ and electrophysiological measures emphasize divergent types of neural processes (Hari 3 and Parkkonen, 2015). While the fast and slow processes share an important degree of spatio4 temporal organization, a considerable proportion of these processes is likely independent. 5 Further, the brain's vascular response is likely coordinated across areas of ICNs (Bright et al., 6 2020), implying that BOLD signal correlations in fMRI may reflect a direct vascular relationship across areas in addition to associated metabolic demands from neural processes as captured by electrophysiological methods. The non-overlapping and complimentary proportion of neural processes in electrophysiology and $\mathrm{fMRI}$ is of practical importance. As a specific example, sourcespace spectral power, amplitude coupling (Engemann et al., 2020) and regional signal variability (standard deviation of amplitude envelopes; Kumral et al., 2020) in M/EEG predict age and neuropsychological scores largely independently of fMRI. These observations motivate revision of the viewpoint that $\mathrm{fMRI}$ and electrophysiology provide different windows onto the same neural processes. Rather, the more exciting view arises that the functional connectome comprises distinct modes of connectivity unfolding in partially non-overlapping spatial and temporal patterns. Depending on their timescale, these patterns dominate the signals in hemodynamic and electrophysiological acquisition methods, respectively.

To conclude, convergence of electrophysiological connectomes with the well-established fMRI connectome implies that connectomes can be successfully derived in source-space $21 \mathrm{MEG} / \mathrm{EEG}$. Conversely, the imperfection of this cross-modal similarity suggests the presence of unique features only observable in one modality. Both of these aspects motivate embracing rather than ignoring ongoing activity and its spatial organization in electrophysiological studies. 
This figure has limited reprint permissions. Please see Figure 2 in original publication
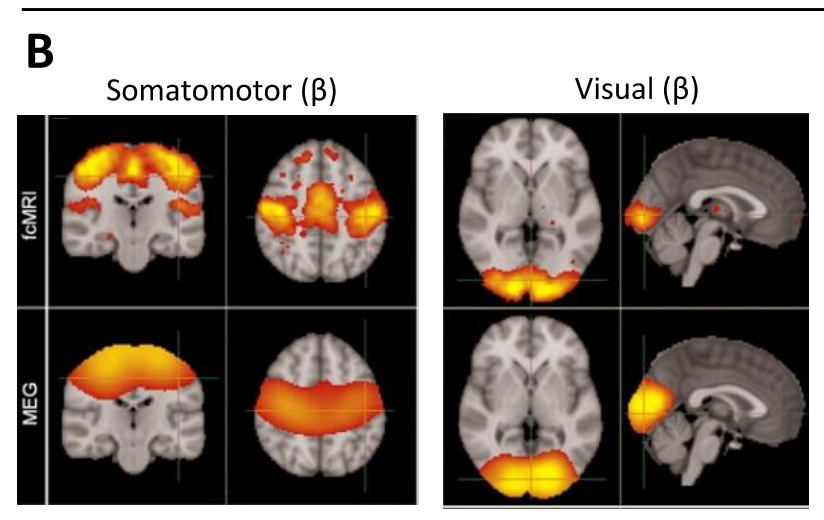

Right Frontoparietal $(\beta)$

Default Mode Network $(\alpha)$

C
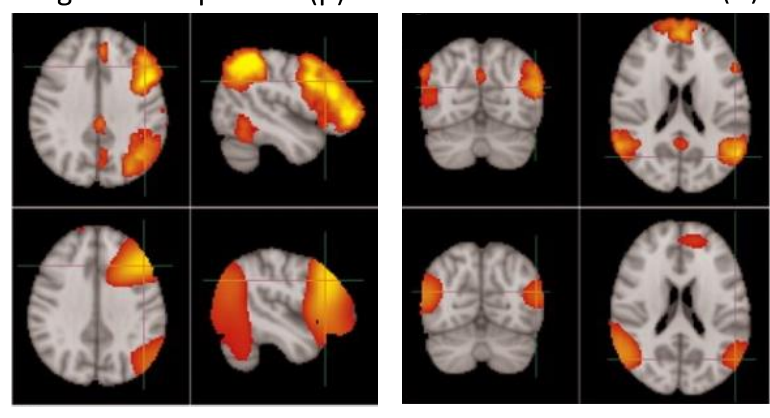

This figure has limited reprint permissions.

Please see Figure 2 in original publication

D

This figure has limited reprint permissions. Please see Figure 2 in original publication (right subplot)

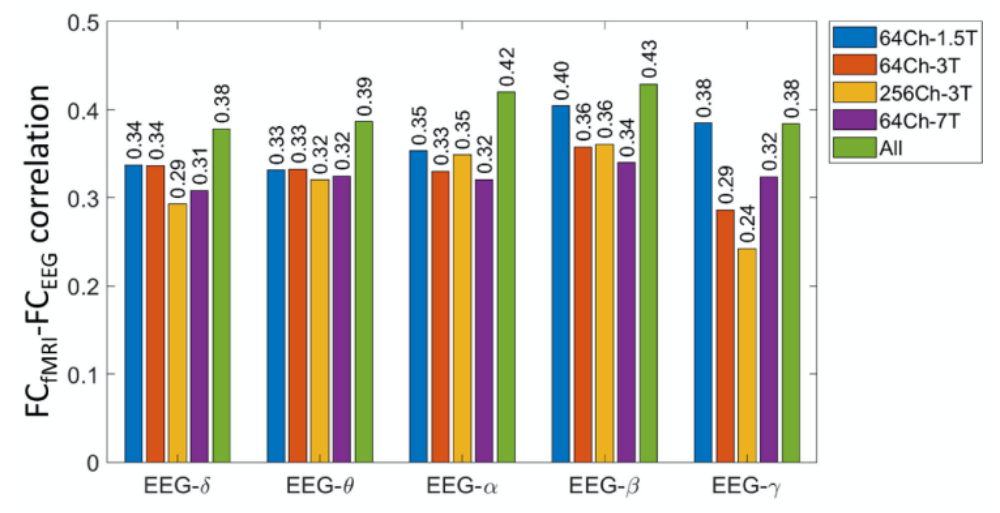



electrophysiological data. A) Sensory and motor ICNs as observed with seed-based connectivity in source-space MEG amplitude coupling. The spectral plot (right) indicates a strong contribution from alpha and beta band oscillations to these intrinsic networks (adapted from Hipp et al., 2012). B) Temporal ICA of band-specific oscillation amplitudes in MEG yields numerous ICNs (four are shown as examples), including sensory/motor as well as higher-order networks. Alpha and especially beta bands captured ICN organization well. A direct comparison between the MEG-derived (bottom row) and the fMRI-derived (toprow) independent component maps demonstrates high spatial similarity (adapted from Brookes et al., 2011). C) Connection-wise connectivity strength is spatially associated between fMRI and intracranial electrophysiology (ECoG amplitude coupling, pooled over patients). The strength of this correlation is around $\sim 0.35$ for all frequency bands (adapted from Betzel et al., 2019). D) A similar spatial association of connection-wise connectivity strength is observed between $\mathrm{FMR}$ and concurrently recorded scalp EEG (phase coupling). The left scatterplot shows an example for the beta band, where each data point is from one connection (region pair) of the connectome averaged across subjects (adapted from Wirsich et al., 2017). This relationship is reproducible at similar effect size across various MRI field strengths (1.5-7T) and EEG densities (64-256 channels) (adapted from Wirsich et al., 2021).

\section{Stability of the intrinsic electrophysiological connectome across cognitive states}

One key characteristic of an intrinsic spatial organization of large-scale connectivity is its relative independence of cognitive context. Such insensitivity to cognitive context has been well quantified in $\mathrm{fMRI}$, demonstrating that task-specific changes to the brain's fMRI-derived timeaveraged connectome organization are small (Cole et al., 2014; Gratton et al., 2018; Hearne et al., 2017). Compared to the aperiodic infra-slow fluctuations of the BOLD signal however, oscillation-based FC is well-positioned to support long-range communication on the rapid timescale of tens to hundreds of milliseconds required for cognitive processes (see Section 3). Could this aptitude result in stronger reconfiguration of electrophysiological FC organization by cognitive context? Electrophysiological connectome investigations such as those discussed above commonly focus on task-free resting state, and few electrophysiological connectome studies have quantitatively compared cognitive states.

One such study analysed daylong iEEG recordings over various levels of arousal and dayto-day activities (Kramer et al., 2011). Amplitude- and phase-coupling derived from periods of 100 $s$ and longer displayed a consistent spatial organization over the diurnal cycle. A related study using sensor-level EEG identified high spatial correlation over different sleep stages and wakefulness for both amplitude- and phase coupling organization (again for periods of $>=100$ s) (Chu et al., 2012). An EEG study in source-space showed that phase-coupling is spatially consistent when calculated over several minutes of different tasks (resting state, video viewing, and flashing gratings) with similar modular organization across frequency bands (Nentwich et al., 2020). Finally, a recent ECoG study contrasted various cognitive states including rest and short (1.5-2.5 s) pre-stimulus and post-stimulus epochs of different tasks (Mostame and Sadaghiani, 2021). Phase- and amplitude-coupling revealed a highly similar, largely state-invariant spatial component across cognitive states. This spatial organization was shared across all frequency bands (though individual bands further exhibited temporally independent FC dynamics that can 45 support frequency-specific information exchange). 
Collectively, these studies suggest that the spatial organization of oscillation-based FC is largely stable over cognitive states (including levels of arousal, resting wakefulness, and task periods with different cognitive demands) i.e., it is primarily intrinsic in nature. This organization is also to a large degree shared across frequency bands. Consequently, dynamic changes in electrophysiological connectomes, including those occurring spontaneously, initiated by task context, or evoked by stimulation should be studied in terms of informative departures from the relatively stable intrinsic organization (cf. Section 3 for methodological aspects).

\section{2) Ongoing electrophysiological activity and connectivity are of behavioural consequence}

Ultimately, the functional significance of the connectome lies in the role it plays in cognition and behaviour. This fundamental role has been established through the observation that electrophysiological connectome organization can be predictive of task behaviour, as we will outline below. Since research into distributed, large-scale connectivity in electrophysiological modalities is rather recent (see Section 3.1), we also discuss more generally the cognitive role of spectral power and phase of ongoing oscillations as they constitute the basis for cross-regional dependencies underlying the large-scale connectome. We close by discussing how these studies may collectively inform about fundamental mechanisms of brain function.

\section{Association of resting state activity and connectivity with behavioural traits across individuals}

Both simple features of the MEG/EEG power spectrum (Da Silva Castanheira et al., 2021; Demuru and Fraschini, 2020) as well as attributes of oscillation-based connectomes (Da Silva Castanheira et al., 2021; Demuru et al., 2017; Nentwich et al., 2020; Sareen et al., 2021) constitute highly subject-specific brain "fingerprints" on the basis of which individuals can be differentiated. Inter-individual differences in electrophysiological attributes, including electrophysiological connectome organization, should thus be considered important to brain function rather than only reflective of noise. This individual specificity suggests an association between such electrophysiological features and behavioural traits. Indeed, long before the era of resting state studies in fMRI, so-called quantitative EEG (QEEG) studies firmly established the wealth of behaviourally relevant information contained in task-free ongoing activity. A long and fruitful history of such studies has shown association between power spectra and related feature derivations with behavioural or clinical traits, and cognitive abilities (Mahjoory et al., 2019; Palva et al., 2013). More recently, distributed phase-coupling at rest has been linked to task performance in the healthy population and aberrations thereof in neurological patients (e.g. Sadaghiani et al., 2019). Turning to source-space EEG, cognitive traits such as IQ have been predicted from phase-coupled connectomes in both resting state and task-based recordings (Nentwich et al., 2020). Interestingly, resting state source-space phase-coupling and amplitudecoupling constitute spatially distinct associations with behavioural task performance (Guggisberg et al., 2015). Further, resting state electrophysiological connectivity not only predicts individuals' perceptual performance but also stimulus-related activation (oscillation power) on tasks (Allaman et al., 2020). 
Behavioural consequences of prestimulus power, phase, and connectivity during task

Complementing the above-described trait-like associations across the population, oscillation-based "states" affect behaviour within individuals. Here, it is important to emphasize that the largely stable core organization of the electrophysiological connectome (extensively discussed in Section 3.1) is complemented by more subtle but behaviourally consequential timevarying changes (cf. Sections 2.3 and 3.3). While the above-described trait-like behavioural associations harness the stable aspect by integrating electrophysiological signals over extensive recordings, the following studies unveil the importance of time-varying dynamics by investigating trial-by-trial variability. An individual's behaviour varies from moment to moment even under comparable external circumstances. A variety of studies suggest that trial-by-trial behavioural variability may covary with prestimulus electrophysiological processes such as prestimulus broadband timeseries (Bode et al., 2012; Britz and Michel, 2010), and the power (Lange et al., 2014; Ruzzoli et al., 2019) as well as phase (Busch et al., 2009; Kayser et al., 2016; Mathewson et al., 2009) of ongoing oscillations at baseline in task-relevant early and higher-order areas. However, the specific nature of the electrophysiological features (e.g. power vs. phase; Ruzzoli et al., 2019; van Diepen et al., 2015) and cognitive processes involved (e.g. perceptual awareness vs. perceptual performance; Benwell et al., 2017) remain debated. A key feature of experimental design shared among these studies is that they maximize behavioural variability (typically measured as accuracy or perceptual choice) by using near-threshold stimuli, ambiguous and illusory stimuli, or conflict-causing stimuli in a cue-free setting. These designs rest on the observation that the less reliable the external information, the more the brain must rely on internal information provided by ongoing activity. Given the often noisy, incomplete, and ambiguous nature of sensory information, the reliance on ongoing activity underlines its putative mechanistic importance in terms of enabling a form of active sensory inference (see discussion of mechanisms below and in Section 4).

Beyond power and phase of oscillations, the coupling of these properties across distributed regions also impacts behaviour. A number of perception studies in MEG/EEG sourcespace have taken a hypothesis-driven approach to selecting a small number of relevant regions activated by the task at hand. Among these task-related regions, prestimulus phase coupling affects perceptual decisions in various task settings and sensory modalities (Hanslmayr et al., 2007; Keil et al., 2014; Rassi et al., 2019). Further, beyond perception tasks, cognitive control processes such as top-down inhibition are affected by prestimulus connectivity states over and above the impact of prestimulus power (Hamm et al., 2012). Finally, the study of prestimulus connectivity has been extended beyond select task-relevant regions to source-localized wholebrain phase coupling and its topology (Weisz et al., 2014). And of course, task-concurrent wholebrain connectomes inform not only about the behavioural impact of prestimulus connectivity, but also of post-stimulus connectivity dynamics (e.g. Favaretto et al., 2021; Hirvonen et al., 2018). The electrophysiological study of large-scale connectomes in task-based experiments is, in our view, particularly underutilised and promising for understanding the functional significance of the statistical variability of connectome measures around a mean core organization. 
Mechanisms underlying behavioural impact

What are the mechanisms driving the association of prestimulus activity and post-stimulus behaviour? A growing body of literature suggests that this association results from the modulation that prestimulus processes exert on stimulus-evoked brain responses. While trial-to-trial variability of evoked responses are rarely acknowledged by most common electrophysiological analysis approaches, such variability is often of similar magnitude to spontaneous fluctuations of ongoing activity. Owing to the alpha rhythm's cyclic modulation of cortical excitability, this rhythm has been extensively studied in this context. It is well established that both prestimulus alpha-band phase and amplitude shape sensory evoked responses (Becker et al., 2008; Fellinger et al., 2011; lemi et al., 2019; Rajagovindan and Ding, 2011), with causal impact on perception (Jaegle and Ro, 2013; Romei et al., 2010). Note that evidence of an influence of connectivity on evoked responses is much less extensive (e.g., Rassi et al., 2019), reinforcing the need to incorporate baseline connectivity in future research.

One prominent suggestion has been that baseline signals represent levels of arousal and alertness/vigilance (Minkwitz et al., 2011), which aligns well with the role of certain oscillations in such general (i.e., unspecific) cognitive functions (Sadaghiani and Kleinschmidt, 2016). Interestingly however, both scalp and intracranial studies using decoding approaches have demonstrated that the influence of baseline activity is not limited to general processes but extends to specific sensory representations. In particular, prestimulus oscillation power (and phase: Li et al., 2020) in sensory areas can be used to directly decode stimulus-specific task-relevant information (Kayser et al., 2016) and modulates post-stimulus decoding of behaviour in a regionby-stimulus specific manner (Li et al., 2020). Interestingly, a recent MEG study suggests that a general process and stimulus-specific information may be concurrently present in different aspects of ongoing activity (Podvalny et al., 2019).

One likely interpretation for the stimulus-specific observations is that prestimulus signals represent intrinsic fluctuations in selective attention, modulating the gain of specific representations. The findings are also in line with another, broader viewpoint regarding the impact on evoked responses and behaviour. According to this viewpoint, this impact results from the possibility that ongoing activity and its spatial organization represent statistical regularities of the world, and that these representations spontaneously reactivate in an iterative manner (Berkes et al., 2011; Sadaghiani and Kleinschmidt, 2013). This interpretation is well-aligned with predictive coding and active inference conceptual frameworks that view perception through the lens of generative models, postulating an indispensable role of prior information in "generating" percepts (Friston et al., 2011; Schroeder et al., 2010). Within the context of this framework, ongoing activity is considered to reflect the neural activity that predicts the causes of sensory inputs as well as the precision or uncertainty of such predictions (Morillon and Baillet, 2017; Sadaghiani et al., 2010). We discuss these aspects further in Section 4.

Irrespective of such a mechanistic purpose, the association of intrinsic oscillations with clinical markers, cognitive abilities, and trial-by-trial behaviour calls for a shift to complement the long-standing research of stimulus-locked and trial-averaged neural processes with the study of ongoing electrophysiological processes and the intrinsic connectome in particular. 


\section{3) Ongoing activity changes dynamically at rapid timescales}

The above discussions on pre-stimulus connectivity and its impact on behaviour and poststimulus processes is the first step towards a dynamic connectivity approach in which the formation and dissolution of networks is mapped in real time as the brain responds during cognition. As outlined in Section 2, electrophysiological approaches have a distinct advantage in this regard, and the evidence presented above suggests that a truly dynamic approach to connectivity - in particular mapping connectivity at the timescale relevant to cognition (i.e., the millisecond timescale) may offer new insights into the underlying enabling functional mechanisms.

The evidence for changes in connectivity over time, even in the resting state, is well established. For example, the sliding window method explained in Section 2 has been used to demonstrate significant temporal fluctuations in electrophysiological connectivity: In early work, DePasquale et al. (2010) demonstrated the use of a 10-s sliding window to elucidate the transient formation of canonical ICNs that had been seen in fMRI. Interestingly, using sliding windows (60 s) in multi-modal recordings, changes in strength of connectivity temporally coincide across concurrent fMRI and EEG (coherence) in all canonical frequency bands and broadly across the cortex (Wirsich et al., 2020). Brookes et al. (2014) showed significant fluctuations of MEG connectivity within the sensorimotor network using a sliding window technique with a window length of $40 \mathrm{~s}$. These observations show how resting state electrophysiological connectivity demonstrates subtle but significant dynamic fluctuations about a central core organization.

Whilst resting state fluctuations of connectivity are apparent, it is sometimes difficult (or even impossible) to link those fluctuations to behaviour. Consequently, a number of attempts have been made to study task evoked changes in electrophysiological connectivity. For example, O'Neill et al. (2015) used a sliding window to demonstrate how electrophysiological connectivity changes with a motor task. This work employed a canonical correlation-based methodology which was able to detect "sub-networks" within the sensorimotor system; in time windows encompassing a button press the authors showed that specific subnetworks within the sensorimotor system were more likely. In this way, both the temporal and spatial variation of network formation was taken into account. A further study by O'Neill et al. (2017b) measured the full connectome matrix using 6-s sliding windows, and demonstrated the formation and dissolution of networks during a Sternberg working memory task. These studies begin to show that a complete dynamic approach to functional connectivity offers new insight into task evoked dynamics. However, for reasons outlined above, especially in Section 2, the utility of the sliding window comes into question, because the inherent timescale of the metrics available is limited to relatively slow fluctuations.

\section{Towards a new generation of functional connectivity}

The limitations of sliding window (and similar) approaches are potentially lifted by the use of the Hidden Markov Model (cf. Section 2.3). In an early paper by Baker et al. (2014), this method was used to uncover the recurrence of brain states that were shown to exist on the time-scale of just a few hundred milliseconds. The identified states each had a distinct spatial signature, which resembled the canonical ICNs previously observed using static connectivity approaches in fMRI and electrophysiology (Figure 3A-B). Post-hoc analysis showed that the classical manifestation of connectivity was underpinned by these rapid fluctuations of network dynamics. In a follow-up 
paper using a time-embedded HMM (able to capture spectral signatures), Vidaurre et al. (2018) showed that canonical ICNs (motor, visual, default-mode) could again be extracted from ongoing electrophysiological data; the default mode was shown to be split in frequency, with parietotemporal projections in alpha and frontal projections in the delta and theta bands. Importantly, once again these networks were shown to modulate on a rapid $(<100 \mathrm{~ms})$ time scale. These initial demonstrations were first to show that the expression of canonical ICNs may evolve on a faster timescale than previously thought. We also note that HMM states may be related to the empirical findings qualified by EEG-microstates, rapidly recurring and broad topographies described in a wide body of literature (Koenig et al., 2005; Lehmann et al., 1998).

The ability to measure millisecond fluctuations in network dynamics begs the question whether the same methodology can be used to understand how ongoing spatio-temporal features of electrophysiological data intersect with a task. Recent work by Higgins and colleagues (Higgins et al., 2021) addressed this question. The authors used HMMs to model network fluctuations during spontaneous memory "replay". Replay is a process by which neural activity associated with specific items is spontaneously initiated in order to consolidate memories. In this context, the "task" is in fact a cognitive process initiated by the brain itself. Replay events have been detected previously in MEG (Liu et al., 2019). Precisely how this process is orchestrated to avoid 'interference' with ongoing cognition was poorly understood. Using an HMM, Higgins and colleagues found that replay events typically occurred selectively during activation of the default mode and parietal alpha networks - two networks known to be associated with inwardly directed attention (see Figure $3 \mathrm{C}$ ). These findings offer the clearest indication yet of how ongoing electrophysiological network activity is modulated dynamically and selectively to support cognitive processing.

In sum, electrophysiology offers the best route to mapping the dynamic connectome with millisecond temporal and high spatial precision. Combined with meaningful measures of neurophysiological interactions (see Section 4), it contributes a better characterisation of resting state task-free data. Further, electrophysiology also contributes to a deeper understanding of the relationship between task-evoked events and ongoing brain activity, in agreement with and complementing fMRI findings about this relationship (Sadaghiani et al., 2010; Sadaghiani and Kleinschmidt, 2013). 


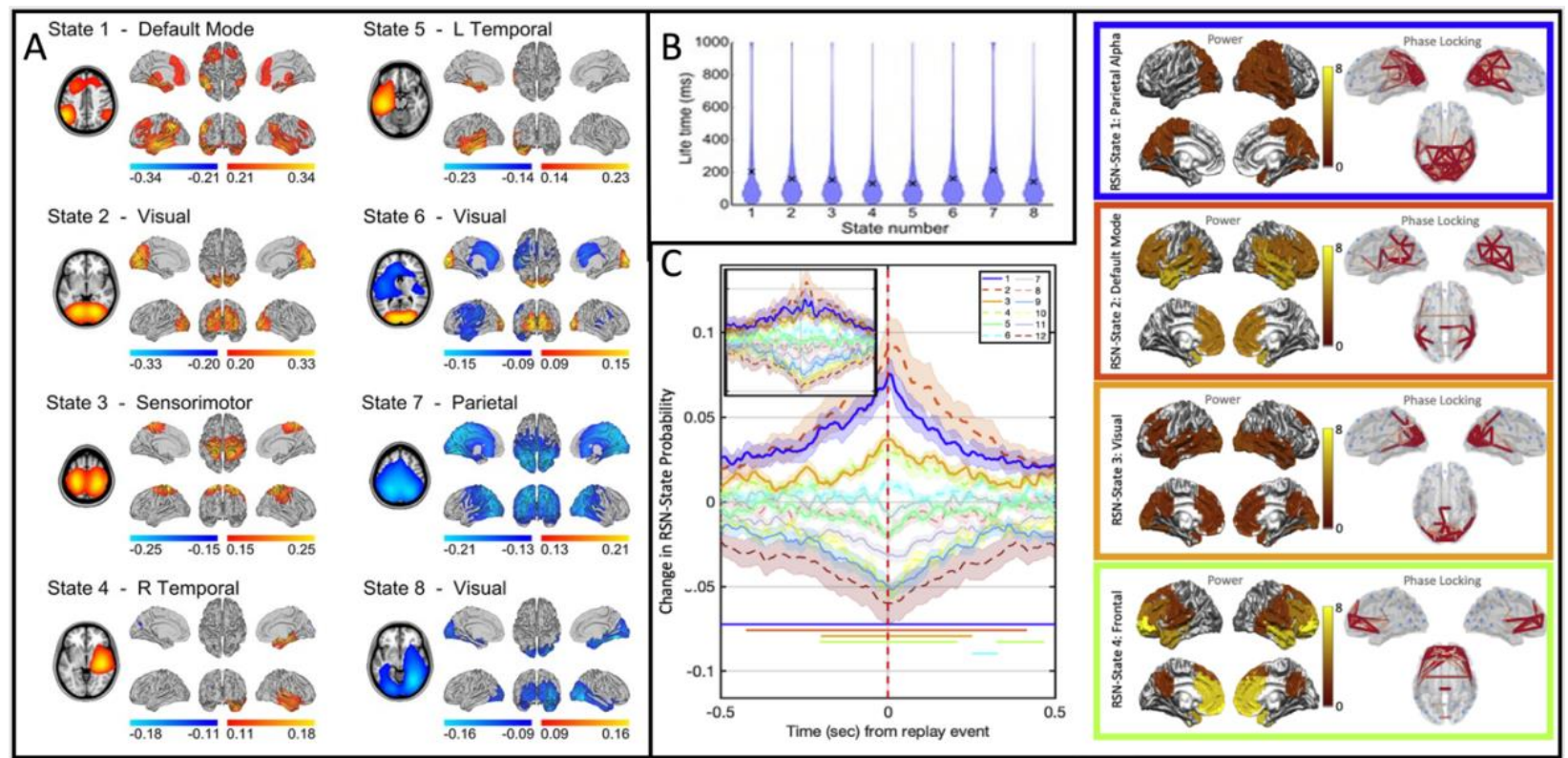

Figure 3: Millisecond dynamics of functional networks. A/B) (Adapted from Baker et al. 2014) A) The spatial signatures of 8 brain states, extracted using a Hidden Markov Model (HMM) applied to resting state MEG data. Each state is determined by a specific topography. These state maps are similar to typical intrinsic connectivity networks (ICNs) commonly observed with fMRI. B) The timescales associated with HMM states are shown in Panel A. Notice that on each state visit, the networks were stable only for short periods of time (100-300 ms) implying that ICNs may fluctuate on a rapid timescale. C) (Adapted from Higgins et al., 2020) "Replay" is the process by which the brain spontaneously revisits recently acquired information in order to e.g., consolidate memories. These replay events are associated with altered probability of occurrence of specific HMM states. The graph on the left shows the change in probability of occurrence of HMM states during a 'replay' event (events at time $t=0$ ). The maps on the right show the four brain networks more likely to be expressed during replay, which prominently included the default mode and parietal alpha networks - both known to be associated with inwardly directed attention.

\section{4) From methods to principles of electrophysiological connectomics, and back.}

\section{1) Caveat: is electrophysiology's fast time resolution too much of a good thing?}

What is the frequency bandwidth of meaningful electrophysiological signals?

The millisecond resolution of electrophysiological signals is their greatest neuroscientific asset. A major result from theory of information is that signal components evolving as fast as half their sampling frequency can convey meaningful contents (Yeung, 2002). Digitization sampling rates of current electrophysiology hardware can be as high as a few tens of $\mathrm{kHz}$ per channel. Does that imply that large-scale brain signal fluctuations as fast as $10 \mathrm{kHz}$ convey bits of information that are meaningful for identifying connectome interactions? In principle yes, but there are important practical considerations.

Every instrument for measuring a certain biological system in a certain environment is susceptible to noise. Noise characterization is often casually overlooked because it is challenging 
or simply impractical. In our field, MEG systems are best equipped for the careful characterization of environmental and instrumental noise conditions. It is indeed considered good practice to run "empty-room" measurements i.e., without a participant present under the MEG sensor array, around each experimental session, to capture noise conditions that may change from session to session (Gross et al., 2013; Hari et al., 2018). Such "dry" data runs help characterize the technical noise floor and its spectro-temporal structure, based on derivatives from power spectrum density (PSD) and empirical sensor covariance estimates (Cohen, 2014).

With EEG, "empty-room" conditions are impractical because electrode signalling requires direct contact with a conducting medium i.e., scalp or an elaborate conductive phantom setup (Baillet et al., 2000). Cut-off frequencies and sampling rates are therefore often defined idiosyncratically and typically set in the realm of hundreds of Hertz. This is neither entirely rigorous nor satisfying, but simply exemplifies how certain aspects of experimental neuroscience are still guided by praxis.

Let us consider data sampled at $1 \mathrm{kHz}$, a range that is commonplace in the field. The maximum frequency accessible for signal analysis is therefore theoretically $500 \mathrm{~Hz}$, and practically about $250-300 \mathrm{~Hz}$, as often imposed by the instrument's additional anti-aliasing hardware filters. A $250-\mathrm{Hz}$ frequency band worth of brain signals represents a vast territory of slow-to-rapid fluctuations to characterize the electrophysiological connectome. From an electrical engineering point of view, electrophysiological data are therefore considered a wide-band signal. There is empirical evidence that scalp and cortical recordings can capture fast (high-frequency) signals that are meaningfully associated with complex human behaviour or clinical symptoms. For instance, neural entrainment by external sensory signals is a powerful experimental approach to mark brain signals of interest responding to sensory stimulation, by boosting specifically their signal-to-noise ratio at fast frequencies up to around $100 \mathrm{~Hz}$ (e.g., visual: (Zhigalov et al., 2019); auditory: (Coffey et al., 2016); somatosensory: (Colon et al., 2012)). Bursts of high-frequency oscillations above $100 \mathrm{~Hz}$ can also be induced by stimulus events (Cimatti et al., 2007) and are generated spontaneously by the epileptic brain (Frauscher et al., 2017). Whether such fast signals play a role in inter-regional communications in brain networks is a matter of active research (Arnulfo et al., 2020; O'Reilly and Elsabbagh, 2021).

In sum, wide-band signals enable rich and varied forms of information channelling. This means that bits of neural information can be conveyed between regions either in parallel via distinct information channels - such as oscillatory signals constrained within narrow frequency bands - and/or via more sophisticated forms of signal encoding, such as phase-amplitude, crossfrequency interactions or all of the above. These considerations have profound implications for producing testable mechanistic hypotheses to comprehend the nature of electrophysiological signal interactions that define the functional connectome.

\section{What is a meaningful measure of connectivity?}

For the reasons outlined above, connectome research in electrophysiology comprises a large toolkit of methods (see Table 2) to measure many possible forms of interactions between complex, time-resolved brain signals. For instance, the Hermes specialized toolbox, FieldTrip, 
and EEGlab's SIFT toolbox feature about two dozen possible measures of connectivity as diverse as Pearson's correlation, variations of phase-locking statistics, different forms of amplitudeamplitude coupling, and directed connectivity measures inspired by econometrics or information theory, etc. (Delorme et al., 2011; Niso et al., 2013; Oostenveld et al., 2010). By contrast, BOLD fluctuations are considerably slower, and fMRI connectome extraction methods rely almost uniquely on measures of time series correlations.

Looking forward, this richness is both a curse and an opportunity for electrophysiology as a field, akin to the years of conundrum on how to best approach inverse modelling for mapping the brain sources of $M E G / E E G$ scalp recordings (Baillet et al., 2001). At the turn of last century, the MEG/EEG community ebulliently (and somewhat confusingly) spawned dozens of source localization and imaging techniques, with standard approaches emerging only very slowly, essentially thanks to the maturation of open-source academic research software (Baillet et al., 2011).

However, with connectivity, the nature of the issue is somewhat different. In source mapping, the source of apparent confusion stemmed from the ill-posed nature of the physics of the electromagnetic inverse problem. In connectivity, ill-posedness is not imposed by the laws of physics, but by the very limited current knowledge of the nature of neurophysiological interactions in large-scale brain networks accessible to electrophysiology.

We have no doubt our field will continue to mature and make progress towards pruning and identifying signal interaction methods that are physiologically meaningful, based on mechanisms vetted, ideally by observations across spatial scales in various experimental preparations and behaving individuals, tested with in-silico computational models, and strengthened by the derivation of causal relations with behaviour and symptoms, using different forms of neuromodulation techniques, for instance.

We envision that open-source software tools are poised to play a role in the maturation and dissemination of good practices in electrophysiological connectivity research via e.g., tutorial documentation and data, practical training workshops, open code debugging and improvements, all promoting and facilitating reproducibility and replicability of connectivity studies.

To encourage the field to steer in that direction, we put forward in the next subsection current leading hypotheses concerning the neurophysiology of signal interactions in large-scale brain networks that are testable via appropriate study designs and accessible to non-invasive electrophysiology.

\section{2) Towards mechanistically driven approaches of electrophysiological connectomics}

\section{Growing beyond the fMRI heritage}

Extending our discussion in Section 3, one entry point to establishing the neurobiological mechanisms of whole-brain connectivity based on electrophysiological signals is to build on current evidence of their concordance with BOLD fluctuations. Some overlap across 
electrophysiological and fMRI-based connectivity can be reasonably expected as both are produced by the same biological system and from the same anatomical scaffold of interconnections. Indeed, we saw in Section 3 the emergence of partial confluence of intrinsic inter-regional signal interactions across data modalities, imposed by the intrinsic, spatial organization of brain networks spanning the modality-specific time scales.

Significant questions remain though: For example, if considerable metabolic resources are spent to generate a wide range of electrophysiological signal components (i.e., frequency bands and modes of connectivity) that are causally related to behaviour (Albouy et al., 2017), with spatial covariations that are remarkably similar across temporal signal time scales, then i) what are the respective functions of these signal components? ii) can we establish how these components interact dynamically, possibly across frequencies? and iii) are these interactions related to connectivity and the expression of brain networks?

Electrophysiological mechanisms of information exchange hold a critical part of the answer to these questions. As we already emphasized above, we shall avoid aiming for full alignment of connectivity across electrophysiology and BOLD, because of the massively different nature of their respective signals. In cross-modal comparisons (cf. Section 3), electrophysiological connectomes can inform about what the BOLD-derived connectome is, but the reverse is not necessarily the case. This is because electrophysiological connectivity entails rapid and temporally precise cross-regional relationships not discernible in fMRI. In this last section of our review, we lay out how combining empirical electrophysiological connectomics with conceptual frameworks could unveil the mechanisms of connectivity causally involved in neural information processing and behaviour.

\section{Advocating for functional connectivity research inspired by physiological mechanisms}

Many electrophysiological connectivity studies are observational and seldom discuss the neurophysiological implications of the connectome effects reported. We emphasize that both the selection of connectivity measures and of the to-be-reported outcomes represent significant decisions that ideally would be informed, to the best of current knowledge, by physiological and mechanistic considerations. Certainly, and depending on the scientific question that motivated a particular study, the scientific value of data-driven and other statistical models must be acknowledged. Yet, their outcomes may not be entirely interpretable in physiological terms.

As reviewed in Section 2, the avenue most extensively explored in electrophysiology so far consists of the assessment of frequency band-specific forms of signal correlations via coherence or amplitude-envelope correlation measures. The rationale for this approach is that slow frequency cycles modulate the firing probability of local neural circuits (e.g., Steriade et al., 1990). Accordingly, regions with time-locked slow-frequency cycles are predisposed to activate in a dynamically coherent fashion. Therefore, these forms of signal similarities may be indicative of two regions working in concert and, by extension, of being nodes of the same functional brain network. 
These basic mechanistic aspects have been best articulated in the conceptual framework of communication through coherence (CTC; Fries, 2015). CTC has contributed a remarkably large body of empirical evidence of effects across the frequency spectrum related to behaviour, in a variety of tasks and electrophysiological preparations (Bastos et al., 2015; Bosman et al., 2012; Michalareas et al., 2016; Schoffelen et al., 2005). Coherence-based methods have also been used to derive whole-brain electrophysiological connectomes (see Section 3). Note that coherence can be high even with substantial phase lags between signals. Hence, as long as lags are stable in time, coherence is, in principle, immune to conduction delays that vary greatly with distance and tract myelination between regions.

Interestingly, alternative approaches that relax the hard assumption of constant phase delays between two network nodes, such as amplitude envelope cross-correlations of bandlimited brain signals (particularly in the beta band $[15,35] \mathrm{Hz}$ ), also successfully produced MEG resting-state connectomes similar to the canonical intrinsic networks of fMRI (Brookes et al., 2011; Figure 2B). However, the amplitude-envelope correlation approach is not as firmly rooted in mechanistic constructs as CTC, which challenges neurophysiological interpretations. More broadly, another point of interrogation concerns the ability of current band-limited models and methods to account for the entirety of the wideband, complex nature of electrophysiological brain signals and their interactions.

We therefore encourage the field to propose and study testable models of brain network functions that are inclusive of a diversity of signal components across the broad frequency spectrum of electrophysiology. The mechanistic concepts should build on the solid body of evidence, rapidly surveyed above, that varied forms of cross-correlations in the phase and/or the amplitude of frequency-specific electrophysiological signals mark interactions between brain regions that may be viewed as dynamical fingerprints of canonical network computations underlying cognitive and other functional processes (Jensen and Colgin, 2007; Siegel et al., 2012). We also advocate for the integrative view that these frequency-specific phenomena are inter-dependent, and that cross-frequency interactions are functionally meaningful to understand brain network functions (Jensen and Colgin, 2007). In essence, the polyrhythmic integrative view of brain networks detailed below is an attempt to reconcile current models and empirical observations and metrics in a holistic fashion. What follows is a proposition and an illustration of how mechanistic models of brain networks may be derived, for the specific purpose of informing electrophysiological connectome studies.

\section{One possible holistic view of functional connectivity enabled by polyrhythmic brain activity}

For this exercise, we begin with the notion surveyed in Section 3 that slower rhythms (delta through alpha) mark the phase of relative excitability of cell assemblies at the mesoscopic regional scale of the human brain. At the other end of the frequency spectrum, gamma and higherfrequency bursts tend to occur in volleys nested at certain preferred phases of these slower rhythms. This is a well-studied phenomenon of cross-frequency interdependencies, which can be revealed by measures such as phase-amplitude coupling (e.g., Canolty and Knight, 2010; cf. Table 2). There is also increasing evidence that these nested gamma cycles may represent timed opportunities for network hubs to register incoming signals in a time-ordered fashion (such as 
sensory inputs or signals from lower-order networks in the hierarchy) by transiently lowering levels of regional inhibition before they are processed by local circuits and relayed further downstream (Lennert et al., 2021; see e.g., Mazaheri et al., 2014).

Building from these elements, we propose that brain regions with aligned cycles of excitability/inhibition - reflected in the phase of the slower delta to alpha frequency ranges - and coherent production of faster activity - reflected in the amplitude of gamma and higher frequency bands - form one functional network (Baillet, 2017; Palva and Palva, 2012). There is both conceptual and empirical evidence that intermediate frequencies in the beta band may play a distinctive role in network signalling (Florin and Baillet, 2015; Schölvinck et al., 2010). Indeed, beta-band oscillations have been proposed to reflect top-down signalling from higher-order brain regions of the central, frontal and prefrontal cortex towards lower-order regions, such as primary sensory areas (Engel and Fries, 2010). In auditory processing for instance, beta bursts issued by frontal regions are time-locked to the expected occurrences of target stimuli, and coupled to the phase of slower oscillations in primary auditory cortices (Morillon and Baillet, 2017; Rimmele et al., 2018). One possible role of these beta bursts may be to reset the phase of slower oscillations (Canavier, 2015) so that their excitability cycles are temporally aligned with the expected occurrences of external stimuli. One putative function of such phase resetting driven by crossfrequency interactions in brain networks would be to optimize the registration of predictable external events at the lowest possible metabolic cost. Beta-band top-down signalling may account, at least in part, for its key role in the extraction of MEG resting-state networks based on amplitude envelope correlations (Brookes et al., 2011; cf. Section 3.1) and individual differentiation with electrophysiological connectome fingerprints (da Silva Castanheira et al., 2021). Further, the fact that beta-band activity is prominent over frontal areas of the human motor system bridges these empirical findings with the theoretical frameworks of active perceptual inference and similar conceptual views (e.g., predictive coding) mentioned in Section 3 (see also, Friston et al., 2011; Schroeder et al., 2010).

The proposed model therefore predicts that the slower frequency cycles of network nodes are temporally aligned with zero phase delay. Such strict alignment over potentially long distances across the brain is supported by computational models (Gollo et al., 2011; Vicente et al., 2008) and has been observed in empirical data (Arnulfo et al., 2020; O'Reilly and Elsabbagh, 2021). For such long-range, zero-lag synchronization to happen it is necessary that some brain regions act as dynamical relays, a role suggested for the cortical and subcortical (e.g., thalamic) hubs of the brain's small-world architecture (Bassett and Bullmore, 2006; Vicente et al., 2008). The dynamic compensation of phase delays between distant network nodes is a fast process that requires only a few oscillatory cycles (Coffey et al., 2021; Cottereau et al., 2011). From an evolutionary perspective, we can speculate that the minimization of such transition periods is critical for quick adjustments of behaviour e.g., when fast motor actions are required to avoid a looming projectile or to register fast-changing language elements in a conversation (Donhauser and Baillet, 2020). Here too, these aspects are consistent with the constructs of active inference and predictive coding, whereby the zeroing of phase delays across the brain may be the manifestation of the correction of prediction errors between lower and higher-level nodes of hierarchical brain networks. 

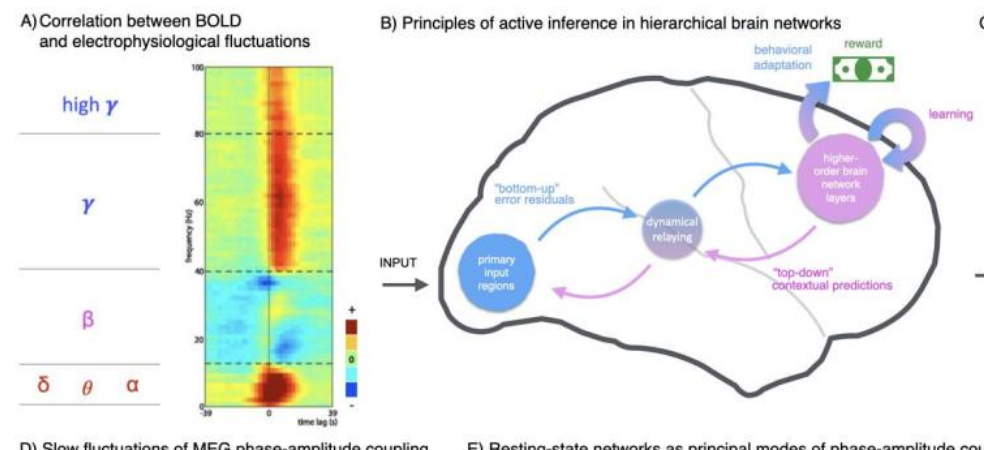

C) A possible biological implementation via coupled, polyrhythmic oscillators future models of the electrophysiological connectome. We re-emphasize that its elements are testable empirically with appropriate study designs via various task conditions, group comparisons (e.g., lesion studies) or neuromodulation techniques. Current experimental validation of these concepts is only fledging, and the models are bound to be updated, refined or refuted. Our intention is to encourage the brain research community to leverage the wealth and complexity of electrophysiological signals with such testable mechanistic models inspired by integrative theoretical frameworks, to advance the elucidation of the nature of brain functions and dysfunctions.

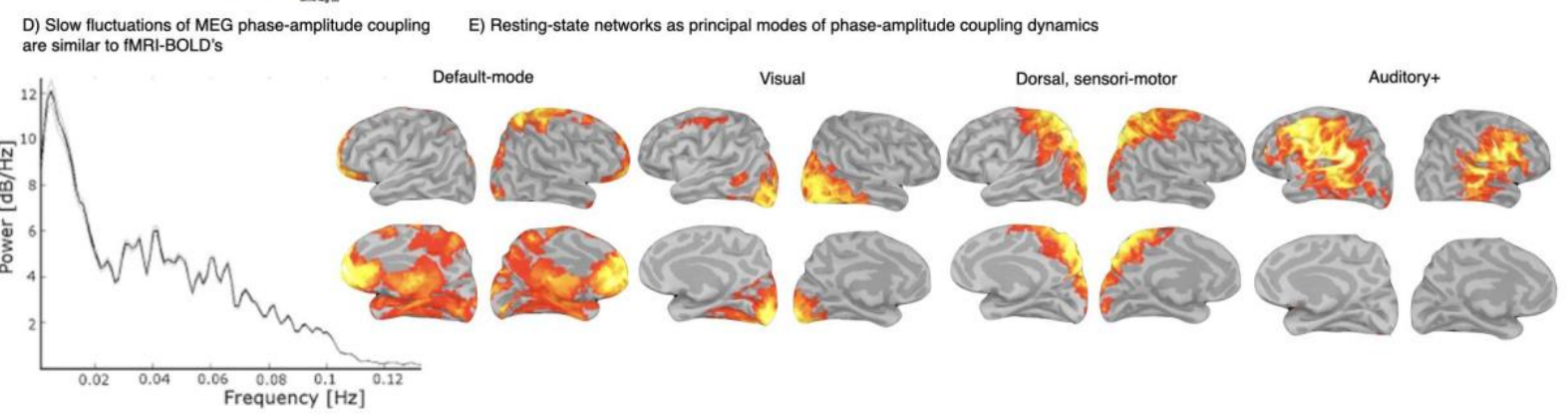

Figure 4 -- Electrophysiological connectomics, from early inspiration by fMRI to testable, mechanistic principles of brain network signalling. A) Cross-correlation between BOLD resting-state and amplitude signal envelopes of typical electrophysiological frequency bands (multimodal non-human primate data collected simultaneously with $\mathrm{fMRI}$ and intracranial EEG). Note the negative cross-correlation of beta-band signals with BOLD, pointing at their possible distinctive role in brain networks, as discussed in Section 4; adapted from Schölvinck et al. (2010). B) Simplified illustration of the basic principles of hierarchical brain networks: exogenous (i.e., external stimulus) signals are registered by low-level, specialized neural circuits (in blue), which also receive endogenous signals from higher-order circuits (in 
pink). These latter are conceptualized as channelling predictive information about input signals. The input circuits compute a form of error signal between these "top-down" predictions and the actual input signal received. The resulting "bottom-up" error signals are relayed directly or indirectly (e.g., via (sub)cortical hub regions as dynamical relays) back to higher-order circuits, where the error signal is registered. This process induces the adaptation of behaviour and the updating of internal predictive models for immediate (reward) and subsequent (learning) behavioural benefits. C) A proposition for the possible biological implementation of these concepts. We illustrate local cross-frequency interactions between low (delta to alpha bands; red sine wave) and higher (gamma to high-gamma bands; blue bursts) frequency signals via e.g., crossfrequency phase-amplitude coupling in regional cell assemblies. Such assemblies are illustrated here as circuits of excitatory (E), fast inhibitory (FI) and slow inhibitory (SI) cells, which can generate such regimes of cross-frequency coupling and are distributed across the brain (Segneri et al., 2020). The illustration also shows beta-band signals as a top-down communication channel (pink). D) Power spectrum of the temporal fluctuations of regional phase-amplitude coupling in the human brain in the resting state. These fluctuations are slow, below $0.1 \mathrm{~Hz}$, a dynamic range compatible with BOLD resting-state fluctuations in fMRI (MEG data from Florin and Baillet, 2015). E) Cross-correlation maps of phase-amplitude coupling fluctuations in the resting brain can be decomposed into spatial modes that are anatomically similar to the typical fMRI resting-state networks (n=12, 5-min resting-state MEG data and methods from Baillet (2017)).

\section{5) Conclusions:}

We started this review and position paper by asking why electrophysiological methods have not been more pervasive in the connectome literature in light of the unique assets that they provide for basic and clinical neuroscience. We outlined two leading contributors to this underrepresentation; one methodological and one conceptual. The methodological challenge rests in estimating electrophysiological connectivity despite confounds due to spurious signal leakage between neuroelectric generators derived from non-invasive electrophysiological recordings. The conceptual challenge arises from the very asset of electrophysiology in terms of richness and complexity of the data. Electrophysiologists are therefore required to make numerous assumptions and methodological choices regarding connectivity measures and timescales.

We believe the electrophysiological connectome studies reviewed here provide convincing evidence that recent technical advances in the field can substantially mitigate the methodological challenges. Specifically, we presented spatial and temporal convergence of connectivity across electrophysiology and structural or functional MRI that don't suffer from the inverse problem. We also highlighted the association of electrophysiological connectivity with behaviour both within and across individuals, and rapid time-varying connectivity dynamics linked to cognitive processes (such as memory consolidation), further supporting the informativeness of sourcelocalized electrophysiological connectivity. Such rapid connectome state changes are largely inaccessible using hemodynamic signals, and this point alone demonstrates the wealth of scientific insight conveyed by electrophysiological connectomics at the timescale of fast cognitive processes, complex behaviour or symptoms.

Addressing the conceptual challenge, we showed how the very nature and richness of electrophysiological signals allow for testing mechanistic hypotheses about cross-region information exchange. In Section 4, we encouraged the field to embrace such a disciplined and 
1 hypothesis-driven approach when taking a leap into the complex territory of electrophysiological 2 connectomics from ongoing brain signals. Specifically, we discussed an in-depth example of one 3 possible conceptual framework that proposes distinct mechanisms subserved by phase-coupling 4 in different frequency bands. This oscillation-based framework exemplifies how the spectral 5 complexity of electrophysiology complements fMRI-based approaches with unique information.

Extending connectomics beyond fMRI that has dominated this field to date is also crucial for another reason. We discussed the possibility that electrophysiological and fMRI connectivity may reflect partially non-overlapping neural and physiological phenomena. Functional MRI is traditionally conceptualized as electrophysiological activity smoothed by the hemodynamic response. However, neural populations and tracts optimized to communicate at fast or slow timescales may be weighted more strongly by electrophysiological and fMRI measures, respectively, and fMRI connectivity may be prone to contributions from vascular demands across regions.

To close, although we have included a dedicated methods section to outline the extensive caution warranted when adopting electrophysiology in human connectivity studies, we argued that shying 18 away from whole-brain electrophysiological connectomics is an opportunity lost for basic and clinical neuroscience research. We therefore hope that the present review and positions motivate a leap of faith to exploit in full the still largely uncharted wealth of distributed ongoing electrophysiological processes of the human brain. 


\section{References}

Albouy, P., Weiss, A., Baillet, S., Zatorre, R.J., 2017. Selective Entrainment of Theta Oscillations in the Dorsal Stream Causally Enhances Auditory Working Memory Performance. Neuron 94, 193-206.e5. https://doi.org/10.1016/j.neuron.2017.03.015

Allaman, L., Mottaz, A., Kleinschmidt, A., Guggisberg, A.G., 2020. Spontaneous Network Coupling Enables Efficient Task Performance without Local Task-Induced Activations. J. Neurosci. 40, 9663-9675. https://doi.org/10.1523/JNEUROSCI.1166-20.2020

Arnulfo, G., Wang, S.H., Myrov, V., Toselli, B., Hirvonen, J., Fato, M.M., Nobili, L., Cardinale, F., Rubino, A., Zhigalov, A., Palva, S., Palva, J.M., 2020. Long-range phase synchronization of high-frequency oscillations in human cortex. Nat. Commun. 11, 5363. https://doi.org/10.1038/s41467-020-18975-8

Baillet, S., 2017. Magnetoencephalography for brain electrophysiology and imaging. Nat. Neurosci. 20, 327-339. https://doi.org/10.1038/nn.4504

Baillet, S., Friston, K., Oostenveld, R., 2011. Academic Software Applications for Electromagnetic Brain Mapping Using MEG and EEG. Comput. Intell. Neurosci. 2011, e972050. https://doi.org/10.1155/2011/972050

Baillet, S., Mosher, J.C., Leahy, R.M., 2001. Electromagnetic brain mapping. IEEE Signal Process. Mag. 18, 14-30. https://doi.org/10.1109/79.962275

Baillet, S., Riera, J.J., Marin, G., Mangin, J.F., Aubert, J., Garnero, L., 2000. Evaluation of inverse methods and head models for EEG source localization using a human skull phantom. Phys. Med. Biol. 46, 77-96. https://doi.org/10.1088/0031-9155/46/1/306

Baker, A.P., Brookes, M.J., Rezek, I.A., Smith, S.M., Behrens, T., Probert Smith, P.J., Woolrich, M., 2014. Fast transient networks in spontaneous human brain activity. eLife 3, e01867. https://doi.org/10.7554/eLife.01867

Bassett, D.S., Bullmore, E., 2006. Small-World Brain Networks. The Neuroscientist 12, 512523. https://doi.org/10.1177/1073858406293182

Bastos, A.M., Schoffelen, J.-M., 2016. A Tutorial Review of Functional Connectivity Analysis Methods and Their Interpretational Pitfalls. Front. Syst. Neurosci. 9, 175. https://doi.org/10.3389/fnsys.2015.00175

Bastos, A.M., Vezoli, J., Fries, P., 2015. Communication through coherence with inter-areal delays. Curr. Opin. Neurobiol., SI: Brain rhythms and dynamic coordination 31, 173-180. https://doi.org/10.1016/j.conb.2014.11.001

Becker, R., Ritter, P., Villringer, A., 2008. Influence of ongoing alpha rhythm on the visual evoked potential. Neurolmage 39, 707-716. https://doi.org/10.1016/j.neuroimage.2007.09.016

Benwell, C.S.Y., Tagliabue, C.F., Veniero, D., Cecere, R., Savazzi, S., Thut, G., 2017. Prestimulus EEG Power Predicts Conscious Awareness But Not Objective Visual Performance. eNeuro 4. https://doi.org/10.1523/ENEURO.0182-17.2017

Berger, H., 1929. Über das Elektrenkephalogramm des Menschen. Arch. Für Psychiatr. Nervenkrankh. 87, 527-570.

Berkes, P., Orbán, G., Lengyel, M., Fiser, J., 2011. Spontaneous Cortical Activity Reveals Hallmarks of an Optimal Internal Model of the Environment. Science 331, 83-87. https://doi.org/10.1126/science.1195870

Betzel, R.F., Medaglia, J.D., Kahn, A.E., Soffer, J., Schonhaut, D.R., Bassett, D.S., 2019. Structural, geometric and genetic factors predict interregional brain connectivity patterns probed by electrocorticography. Nat. Biomed. Eng. 1. https://doi.org/10.1038/s41551 019-0404-5 
Bode, S., Sewell, D.K., Lilburn, S., Forte, J.D., Smith, P.L., Stahl, J., 2012. Predicting Perceptual Decision Biases from Early Brain Activity. J. Neurosci. 32, 12488-12498. https://doi.org/10.1523/JNEUROSCI.1708-12.2012

Bosman, C.A., Schoffelen, J.-M., Brunet, N., Oostenveld, R., Bastos, A.M., Womelsdorf, T., Rubehn, B., Stieglitz, T., De Weerd, P., Fries, P., 2012. Attentional Stimulus Selection through Selective Synchronization between Monkey Visual Areas. Neuron 75, 875-888. https://doi.org/10.1016/j.neuron.2012.06.037

Boto, E., Hill, R.M., Rea, M., Holmes, N., Seedat, Z.A., Leggett, J., Shah, V., Osborne, J., Bowtell, R., Brookes, M.J., 2021. Measuring functional connectivity with wearable MEG. Neurolmage 230, 117815. https://doi.org/10.1016/j.neuroimage.2021.117815

Boto, E., Seedat, Z.A., Holmes, N., Leggett, J., Hill, R.M., Roberts, G., Shah, V., Fromhold, T.M., Mullinger, K.J., Tierney, T.M., Barnes, G.R., Bowtell, R., Brookes, M.J., 2019. Wearable neuroimaging: Combining and contrasting magnetoencephalography and electroencephalography. Neurolmage 201, 116099. https://doi.org/10.1016/j.neuroimage.2019.116099

Breakspear, M., Williams, L.M., Stam, C.J., 2004. A Novel Method for the Topographic Analysis of Neural Activity Reveals Formation and Dissolution of 'Dynamic Cell Assemblies.' J. Comput. Neurosci. 16, 49-68. https://doi.org/10.1023/B:JCNS.0000004841.66897.7d

Bright, M.G., Whittaker, J.R., Driver, I.D., Murphy, K., 2020. Vascular physiology drives functional brain networks. Neurolmage 217, 116907. https://doi.org/10.1016/j.neuroimage.2020.116907

Britz, J., Michel, C.M., 2010. Errors can be related to pre-stimulus differences in ERP topography and their concomitant sources. Neurolmage 49, 2774-2782. https://doi.org/10.1016/j.neuroimage.2009.10.033

Brookes, M.J., Woolrich, M., Luckhoo, H., Price, D., Hale, J.R., Stephenson, M.C., Barnes, G.R., Smith, S.M., Morris, P.G., 2011. Investigating the electrophysiological basis of resting state networks using magnetoencephalography. Proc. Natl. Acad. Sci. 108, 16783-16788. https://doi.org/10.1073/pnas.1112685108

Busch, N.A., Dubois, J., VanRullen, R., 2009. The Phase of Ongoing EEG Oscillations Predicts Visual Perception. J Neurosci 29, 7869-7876. https://doi.org/10.1523/JNEUROSCI.0113-09.2009

Cabral, J., Luckhoo, H., Woolrich, M., Joensson, M., Mohseni, H., Baker, A., Kringelbach, M.L., Deco, G., 2014. Exploring mechanisms of spontaneous functional connectivity in MEG: How delayed network interactions lead to structured amplitude envelopes of band-pass filtered oscillations. Neurolmage 90, 423-435. https://doi.org/10.1016/j.neuroimage.2013.11.047

Canavier, C.C., 2015. Phase-resetting as a tool of information transmission. Curr. Opin. Neurobiol., SI: Brain rhythms and dynamic coordination 31, 206-213. https://doi.org/10.1016/j.conb.2014.12.003

Canolty, R.T., Knight, R.T., 2010. The functional role of cross-frequency coupling. Trends Cogn. Sci. 14, 506-515. https://doi.org/10.1016/j.tics.2010.09.001

Cao, J., Zhao, Y., Shan, X., Wei, H., Guo, Y., Chen, L., Erkoyuncu, J.A., Sarrigiannis, P.G., 2021. Brain functional and effective connectivity based on electroencephalography recordings: A review. Hum. Brain Mapp. n/a. https://doi.org/10.1002/hbm.25683

Chang, C., Glover, G.H., 2010. Time-frequency dynamics of resting-state brain connectivity measured with fMRI. Neurolmage 50, 81-98. https://doi.org/10.1016/j.neuroimage.2009.12.011

Chu, C., Tanaka, N., Diaz, J., Edlow, B., Wu, O., Hämäläinen, M., Stufflebeam, S., Cash, S., Kramer, MA., 2015. EEG functional connectivity is partially predicted by underlying white matter connectivity. Neurolmage 108, 23-33. https://doi.org/10.1016/j.neuroimage.2014.12.033 
Chu, C.J., Kramer, M.A., Pathmanathan, J., Bianchi, M.T., Westover, M.B., Wizon, L., Cash, S.S., 2012. Emergence of Stable Functional Networks in Long-Term Human Electroencephalography. J. Neurosci. 32, 2703-2713. https://doi.org/10.1523/JNEUROSCI.5669-11.2012

Cimatti, Z., P Schwartz, D., Bourdain, F., Meunier, S., Bleton, J.-P., Vidailhet, M., Renault, B., Garnero, L., 2007. Time-frequency analysis reveals decreased high-frequency oscillations in writer's cramp. Brain 130, 198-205. https://doi.org/10.1093/brain/awl259

Coffey, E.B.J., Arseneau-Bruneau, I., Zhang, X., Baillet, S., Zatorre, R.J., 2021. Oscillatory entrainment of the Frequency Following Response in auditory cortical and subcortical structures. J. Neurosci. https://doi.org/10.1523/JNEUROSCI.2313-20.2021

Coffey, E.B.J., Herholz, S.C., Chepesiuk, A.M.P., Baillet, S., Zatorre, R.J., 2016. Cortical contributions to the auditory frequency-following response revealed by MEG. Nat. Commun. 7, 11070. https://doi.org/10.1038/ncomms11070

Cohen, D., 1972. Magnetoencephalography: Detection of the Brain's Electrical Activity with a Superconducting Magnetometer. Science 175, 664-666. https://doi.org/10.1126/science.175.4022.664

Cohen, M.X., 2014. Analyzing Neural Time Series Data. The MIT Press.

Colclough, G.L., Brookes, M.J., Smith, S.M., Woolrich, M.W., 2015. A symmetric multivariate leakage correction for MEG connectomes. Neurolmage 117, 439-448. https://doi.org/10.1016/j.neuroimage.2015.03.071

Cole, M.W., Bassett, D.S., Power, J.D., Braver, T.S., Petersen, S.E., 2014. Intrinsic and TaskEvoked Network Architectures of the Human Brain. Neuron 83, 238-251. https://doi.org/10.1016/j.neuron.2014.05.014

Colon, E., Legrain, V., Mouraux, A., 2012. Steady-state evoked potentials to study the processing of tactile and nociceptive somatosensory input in the human brain. Neurophysiol. Clin. Neurophysiol. 42, 315-323. https://doi.org/10.1016/j.neucli.2012.05.005

Cottereau, B., Lorenceau, J., Gramfort, A., Clerc, M., Thirion, B., Baillet, S., 2011. Phase delays within visual cortex shape the response to steady-state visual stimulation. Neurolmage 54, 1919-1929. https://doi.org/10.1016/j.neuroimage.2010.10.004

Da Silva Castanheira, J., Orozco, H.D., Misic, B., Baillet, S., 2021. MEG, myself, and I: individual identification from neurophysiological brain activity (preprint). Neuroscience. https://doi.org/10.1101/2021.02.18.431803

da Silva Castanheira, J., Orozco Perez, H.D., Misic, B., Baillet, S., 2021. Brief segments of neurophysiological activity enable individual differentiation. Nat. Commun. 12, 5713. https://doi.org/10.1038/s41467-021-25895-8

de Pasquale, F., Penna, S.D., Snyder, A.Z., Lewis, C., Mantini, D., Marzetti, L., Belardinelli, P., Ciancetta, L., Pizzella, V., Romani, G.L., Corbetta, M., 2010. Temporal dynamics of spontaneous MEG activity in brain networks. Proc. Natl. Acad. Sci. 107, 6040-6045. https://doi.org/10.1073/pnas.0913863107

Deligianni, F., Carmichael, D.W., Zhang, G.H., Clark, C.A., Clayden, J.D., 2016. NODDI and Tensor-Based Microstructural Indices as Predictors of Functional Connectivity. PLOS ONE 11, e0153404. https://doi.org/10.1371/journal.pone.0153404

Delorme, A., Mullen, T., Kothe, C., Akalin Acar, Z., Bigdely-Shamlo, N., Vankov, A., Makeig, S., 2011. EEGLAB, SIFT, NFT, BCILAB, and ERICA: New Tools for Advanced EEG Processing. Comput. Intell. Neurosci. 2011, e130714. https://doi.org/10.1155/2011/130714

Demuru, M., Fraschini, M., 2020. EEG fingerprinting: Subject-specific signature based on the aperiodic component of power spectrum. Comput. Biol. Med. 120, 103748. https://doi.org/10.1016/j.compbiomed.2020.103748 
Demuru, M., Gouw, A.A., Hillebrand, A., Stam, C.J., Dijk, B.W. van, Scheltens, P., Tijms, B.M., Konijnenberg, E., Kate, M. ten, Braber, A. den, Smit, D.J.A., Boomsma, D.I., Visser, P.J., 2017. Functional and effective whole brain connectivity using magnetoencephalography to identify monozygotic twin pairs. Sci. Rep. 7, 9685. https://doi.org/10.1038/s41598017-10235-y

Despouy, E., Curot, J., Reddy, L., Nowak, L.G., Deudon, M., Sol, J.-C., Lotterie, J.-A., Denuelle, M., Maziz, A., Bergaud, C., Thorpe, S.J., Valton, L., Barbeau, E.J., 2020. Recording local field potential and neuronal activity with tetrodes in epileptic patients. J. Neurosci. Methods 341, 108759. https://doi.org/10.1016/j.jneumeth.2020.108759

Donhauser, P.W., Baillet, S., 2020. Two Distinct Neural Timescales for Predictive Speech Processing. Neuron 105, 385-393.e9. https://doi.org/10.1016/j.neuron.2019.10.019

Driver, J., Blankenburg, F., Bestmann, S., Vanduffel, W., Ruff, C.C., 2009. Concurrent brainstimulation and neuroimaging for studies of cognition. Trends Cogn. Sci. 13, 319-327. https://doi.org/10.1016/j.tics.2009.04.007

Engel, A.K., Fries, P., 2010. Beta-band oscillations -- signalling the status quo? Curr. Opin. Neurobiol. 20, 156-165. https://doi.org/10.1016/j.conb.2010.02.015

Engel, A.K., Gerloff, C., Hilgetag, C.C., Nolte, G., 2013. Intrinsic Coupling Modes: Multiscale Interactions in Ongoing Brain Activity. Neuron 80, 867-886. https://doi.org/10.1016/j.neuron.2013.09.038

Engemann, D.A., Kozynets, O., Sabbagh, D., Lemaître, G., Varoquaux, G., Liem, F., Gramfort, A., 2020. Combining magnetoencephalography with magnetic resonance imaging enhances learning of surrogate-biomarkers. eLife 9, e54055. https://doi.org/10.7554/eLife.54055

Engemann, D.A., Raimondo, F., King, J.-R., Rohaut, B., Louppe, G., Faugeras, F., Annen, J., Cassol, H., Gosseries, O., Fernandez-Slezak, D., Laureys, S., Naccache, L., Dehaene, S., Sitt, J.D., 2018. Robust EEG-based cross-site and cross-protocol classification of states of consciousness. Brain 141, 3179-3192. https://doi.org/10.1093/brain/awy251

Favaretto, C., Spadone, S., Sestieri, C., Betti, V., Cenedese, A., Della Penna, S., Corbetta, M., 2021. Multi-band MEG signatures of BOLD connectivity reorganization during visuospatial attention. Neurolmage 230, 117781. https://doi.org/10.1016/j.neuroimage.2021.117781

Fellinger, R., Klimesch, W., Gruber, W., Freunberger, R., Doppelmayr, M., 2011. Pre-stimulus alpha phase-alignment predicts P1-amplitude. Brain Res. Bull. 85, 417-423. https://doi.org/10.1016/j.brainresbull.2011.03.025

Finger, H., Bönstrup, M., Cheng, B., Messé, A., Hilgetag, C., Thomalla, G., Gerloff, C., König, P., 2016. Modeling of Large-Scale Functional Brain Networks Based on Structural Connectivity from DTI: Comparison with EEG Derived Phase Coupling Networks and Evaluation of Alternative Methods along the Modeling Path. PLOS Comput. Biol. 12, e1005025. https://doi.org/10.1371/journal.pcbi.1005025

Florin, E., Baillet, S., 2018. Commentary: Evaluation of Phase-Amplitude Coupling in Resting State Magnetoencephalographic Signals: Effect of Surrogates and Evaluation Approach. Front. Comput. Neurosci. 12, 26. https://doi.org/10.3389/fncom.2018.00026

Florin, E., Baillet, S., 2015. The brain's resting-state activity is shaped by synchronized crossfrequency coupling of neural oscillations. Neurolmage 111, 26-35. https://doi.org/10.1016/j.neuroimage.2015.01.054

Frauscher, B., Bartolomei, F., Kobayashi, K., Cimbalnik, J., Klooster, M.A. van 't, Rampp, S., Otsubo, H., Höller, Y., Wu, J.Y., Asano, E., Engel, J., Kahane, P., Jacobs, J., Gotman, J., 2017. High-frequency oscillations: The state of clinical research. Epilepsia 58, 13161329. https://doi.org/10.1111/epi.13829 
Fried, I., MacDonald, K.A., Wilson, C.L., 1997. Single Neuron Activity in Human Hippocampus and Amygdala during Recognition of Faces and Objects. Neuron 18, 753-765. https://doi.org/10.1016/S0896-6273(00)80315-3

Fries, P., 2015. Rhythms for Cognition: Communication through Coherence. Neuron 88, $220-$ 235. https://doi.org/10.1016/j.neuron.2015.09.034

Friston, K., Mattout, J., Kilner, J., 2011. Action understanding and active inference. Biol. Cybern. 104, 137-160. https://doi.org/10.1007/s00422-011-0424-z

Glasser, M.F., Smith, S.M., Marcus, D.S., Andersson, J.L.R., Auerbach, E.J., Behrens, T.E.J., Coalson, T.S., Harms, M.P., Jenkinson, M., Moeller, S., Robinson, E.C., Sotiropoulos, S.N., Xu, J., Yacoub, E., Ugurbil, K., Essen, D.C.V., 2016. The Human Connectome Project's neuroimaging approach. Nat. Neurosci. 19, 1175-1187. https://doi.org/10.1038/nn.4361

Gollo, L.L., Mirasso, C.R., Atienza, M., Crespo-Garcia, M., Cantero, J.L., 2011. Theta Band Zero-Lag Long-Range Cortical Synchronization via Hippocampal Dynamical Relaying. PLOS ONE 6, e17756. https://doi.org/10.1371/journal.pone.0017756

Gratton, C., Laumann, T.O., Nielsen, A.N., Greene, D.J., Gordon, E.M., Gilmore, A.W., Nelson, S.M., Coalson, R.S., Snyder, A.Z., Schlaggar, B.L., Dosenbach, N.U.F., Petersen, S.E., 2018. Functional Brain Networks Are Dominated by Stable Group and Individual Factors, Not Cognitive or Daily Variation. Neuron 98, 439-452.e5. https://doi.org/10.1016/j.neuron.2018.03.035

Gross, J., Baillet, S., Barnes, G.R., Henson, R.N., Hillebrand, A., Jensen, O., Jerbi, K., Litvak, V., Maess, B., Oostenveld, R., Parkkonen, L., Taylor, J.R., van Wassenhove, V., Wibral, M., Schoffelen, J.-M., 2013. Good practice for conducting and reporting MEG research. Neurolmage 65, 349-363. https://doi.org/10.1016/j.neuroimage.2012.10.001

Guggisberg, A.G., Rizk, S., Ptak, R., Di Pietro, M., Saj, A., Lazeyras, F., Lovblad, K.-O., Schnider, A., Pignat, J.-M., 2015. Two Intrinsic Coupling Types for Resting-State Integration in the Human Brain. Brain Topogr. 28, 318-329. https://doi.org/10.1007/s10548-014-0394-2

Hacker, C.D., Snyder, A.Z., Pahwa, M., Corbetta, M., Leuthardt, E.C., 2017. Frequency-specific electrophysiologic correlates of resting state fMRI networks. Neurolmage 149, 446-457. https://doi.org/10.1016/j.neuroimage.2017.01.054

Hamm, J.P., Dyckman, K.A., McDowell, J.E., Clementz, B.A., 2012. Pre-Cue Fronto-Occipital Alpha Phase and Distributed Cortical Oscillations Predict Failures of Cognitive Control. J. Neurosci. 32, 7034-7041. https://doi.org/10.1523/JNEUROSCI.5198-11.2012

Hanslmayr, S., Aslan, A., Staudigl, T., Klimesch, W., Herrmann, C.S., Bäuml, K.-H., 2007. Prestimulus oscillations predict visual perception performance between and within subjects. Neurolmage 37, 1465-1473. https://doi.org/10.1016/j.neuroimage.2007.07.011

Hari, R., Baillet, S., Barnes, G., Burgess, R., Forss, N., Gross, J., Hämäläinen, M., Jensen, O., Kakigi, R., Mauguière, F., Nakasato, N., Puce, A., Romani, G.-L., Schnitzler, A., Taulu, S., 2018. IFCN-endorsed practical guidelines for clinical magnetoencephalography (MEG). Clin. Neurophysiol. 129, 1720-1747. https://doi.org/10.1016/j.clinph.2018.03.042

Hari, R., Parkkonen, L., 2015. The brain timewise: how timing shapes and supports brain function. Philos. Trans. R. Soc. B Biol. Sci. 370, 20140170. https://doi.org/10.1098/rstb.2014.0170

He, B.J., Snyder, A.Z., Zempel, J.M., Smyth, M.D., Raichle, M.E., 2008. Electrophysiological correlates of the brain's intrinsic large-scale functional architecture. Proc. Natl. Acad. Sci. U. S. A. 105, 16039-16044. https://doi.org/10.1073/pnas.0807010105

Hearne, L.J., Cocchi, L., Zalesky, A., Mattingley, J.B., 2017. Reconfiguration of Brain Network Architectures between Resting-State and Complexity-Dependent Cognitive Reasoning. J. Neurosci. 37, 8399-8411. https://doi.org/10.1523/JNEUROSCI.0485-17.2017 
Helfrich, R.F., Knepper, H., Nolte, G., Sengelmann, M., König, P., Schneider, T.R., Engel, A.K., 2016. Spectral fingerprints of large-scale cortical dynamics during ambiguous motion perception. Hum. Brain Mapp. 37, 4099-4111. https://doi.org/10.1002/hbm.23298

Henry, J.C., 2006. Electroencephalography: Basic Principles, Clinical Applications, and Related Fields, Fifth Edition. Neurology 67, 2092-2092-a. https://doi.org/10.1212/01.wnl.0000243257.85592.9a

Higgins, C., Liu, Y., Vidaurre, D., Kurth-Nelson, Z., Dolan, R., Behrens, T., Woolrich, M., 2021. Replay bursts in humans coincide with activation of the default mode and parietal alpha networks. Neuron 109, 882-893.e7. https://doi.org/10.1016/j.neuron.2020.12.007

Hipp, J.F., Hawellek, D.J., Corbetta, M., Siegel, M., Engel, A.K., 2012. Large-scale cortical correlation structure of spontaneous oscillatory activity. Nat. Neurosci. 15, 884-890. https://doi.org/10.1038/nn.3101

Hipp, J.F., Siegel, M., 2015. BOLD fMRI Correlation Reflects Frequency-Specific Neuronal Correlation. Curr. Biol. 25, 1368-1374. https://doi.org/10.1016/j.cub.2015.03.049

Hirvonen, J., Monto, S., Wang, S.H., Palva, J.M., Palva, S., 2018. Dynamic large-scale network synchronization from perception to action. Netw. Neurosci. 2, 442-463. https://doi.org/10.1162/netn_a_00039

Hunyadi, B., Woolrich, M., Quinn, A., Vidaurre, D., De Vos, M., 2018. A dynamic system of brain networks revealed by fast transient EEG fluctuations and their fMRI correlates. Neurolmage. https://doi.org/10.1016/j.neuroimage.2018.09.082

lemi, L., Busch, N.A., Laudini, A., Haegens, S., Samaha, J., Villringer, A., Nikulin, V.V., 2019. Multiple mechanisms link prestimulus neural oscillations to sensory responses. eLife 8 , e43620. https://doi.org/10.7554/eLife.43620

Jaegle, A., Ro, T., 2013. Direct Control of Visual Perception with Phase-specific Modulation of Posterior Parietal Cortex. J. Cogn. Neurosci. 26, 422-432. https://doi.org/10.1162/jocn_a_00494

Jensen, O., Colgin, L.L., 2007. Cross-frequency coupling between neuronal oscillations. Trends Cogn. Sci. 11, 267-269. https://doi.org/10.1016/j.tics.2007.05.003

Jeromin, A., Bowser, R., 2017. Biomarkers in Neurodegenerative Diseases, in: Beart, P., Robinson, M., Rattray, M., Maragakis, N.J. (Eds.), Neurodegenerative Diseases: Pathology, Mechanisms, and Potential Therapeutic Targets, Advances in Neurobiology. Springer International Publishing, Cham, pp. 491-528. https://doi.org/10.1007/978-3319-57193-5_20

Kayser, S.J., McNair, S.W., Kayser, C., 2016. Prestimulus influences on auditory perception from sensory representations and decision processes. Proc. Natl. Acad. Sci. 113, 48424847. https://doi.org/10.1073/pnas.1524087113

Keil, J., Müller, N., Hartmann, T., Weisz, N., 2014. Prestimulus Beta Power and Phase Synchrony Influence the Sound-Induced Flash Illusion. Cereb. Cortex 24, 1278-1288. https://doi.org/10.1093/cercor/bhs409

Keller, C.J., Bickel, S., Honey, C.J., Groppe, D.M., Entz, L., Craddock, R.C., Lado, F.A., Kelly, C., Milham, M., Mehta, A.D., 2013. Neurophysiological Investigation of Spontaneous Correlated and Anticorrelated Fluctuations of the BOLD Signal. J. Neurosci. 33, 63336342. https://doi.org/10.1523/JNEUROSCI.4837-12.2013

Kim, C.K., Adhikari, A., Deisseroth, K., 2017. Integration of optogenetics with complementary methodologies in systems neuroscience. Nat. Rev. Neurosci. 18, 222-235. https://doi.org/10.1038/nrn.2017.15

Kim, K., 2016. A network approach for modulating memory processes via direct and indirect brain stimulation: Toward a causal approach for the neural basis of memory. Neurobiol. Learn. Mem. 16. 
Ko, A.L., Weaver, K.E., Hakimian, S., Ojemann, J.G., 2013. Identifying Functional Networks Using Endogenous Connectivity in Gamma Band Electrocorticography. Brain Connect. 3, 491-502. https://doi.org/10.1089/brain.2013.0157

Koenig, T., Studer, D., Hubl, D., Melie, L., Strik, W. k, 2005. Brain connectivity at different timescales measured with EEG. Philos. Trans. R. Soc. B Biol. Sci. 360, 1015-1024. https://doi.org/10.1098/rstb.2005.1649

Kramer, M.A., Eden, U.T., Lepage, K.Q., Kolaczyk, E.D., Bianchi, M.T., Cash, S.S., 2011. Emergence of Persistent Networks in Long-Term Intracranial EEG Recordings. J. Neurosci. 31, 15757-15767. https://doi.org/10.1523/JNEUROSCI.2287-11.2011

Kucyi, A., Schrouff, J., Bickel, S., Foster, B.L., Shine, J.M., Parvizi, J., 2018. Intracranial electrophysiology reveals reproducible intrinsic functional connectivity within human brain networks. J. Neurosci. 0217-18. https://doi.org/10.1523/JNEUROSCI.021718.2018

Kujala, J., Gross, J., Salmelin, R., 2008. Localization of correlated network activity at the cortical level with MEG. Neurolmage 39, 1706-1720. https://doi.org/10.1016/j.neuroimage.2007.10.042

Kumral, D., Şansal, F., Cesnaite, E., Mahjoory, K., Al, E., Gaebler, M., Nikulin, V.V., Villringer, A., 2020. BOLD and EEG signal variability at rest differently relate to aging in the human brain. Neurolmage 207, 116373. https://doi.org/10.1016/j.neuroimage.2019.116373

Lange, J., Keil, J., Schnitzler, A., van Dijk, H., Weisz, N., 2014. The role of alpha oscillations for illusory perception. Behav. Brain Res. 271, 294-301. https://doi.org/10.1016/j.bbr.2014.06.015

Lehmann, D., Strik, W.K., Henggeler, B., Koenig, T., Koukkou, M., 1998. Brain electric microstates and momentary conscious mind states as building blocks of spontaneous thinking: I. Visual imagery and abstract thoughts. Int. J. Psychophysiol. 29, 1-11. https://doi.org/10.1016/S0167-8760(97)00098-6

Lennert, T., Samiee, S., Baillet, S., 2021. Coupled oscillations enable rapid temporal recalibration to audiovisual asynchrony. Commun. Biol. 4, 1-12. https://doi.org/10.1038/s42003-021-02087-0

Li, Y., Ward, M.J., Richardson, R.M., G'Sell, M., Ghuman, A.S., 2020. Endogenous activity modulates stimulus and circuit-specific neural tuning and predicts perceptual behavior. Nat. Commun. 11, 4014. https://doi.org/10.1038/s41467-020-17729-w

Liu, Y., Dolan, R.J., Kurth-Nelson, Z., Behrens, T.E.J., 2019. Human Replay Spontaneously Reorganizes Experience. Cell 178, 640-652.e14. https://doi.org/10.1016/j.cell.2019.06.012

Mahjoory, K., Cesnaite, E., Hohlefeld, F.U., Villringer, A., Nikulin, V.V., 2019. Power and temporal dynamics of alpha oscillations at rest differentiate cognitive performance involving sustained and phasic cognitive control. Neurolmage 188, 135-144. https://doi.org/10.1016/j.neuroimage.2018.12.001

Mathewson, K.E., Gratton, G., Fabiani, M., Beck, D.M., Ro, T., 2009. To See or Not to See: Prestimulus \{alpha\} Phase Predicts Visual Awareness. J Neurosci 29, 2725-2732. https://doi.org/10.1523/JNEUROSCI.3963-08.2009

Mazaheri, A., van Schouwenburg, M.R., Dimitrijevic, A., Denys, D., Cools, R., Jensen, O., 2014. Region-specific modulations in oscillatory alpha activity serve to facilitate processing in the visual and auditory modalities. Neurolmage 87, 356-362. https://doi.org/10.1016/j.neuroimage.2013.10.052

McCormick, D.A., Contreras, D., 2001. On The Cellular and Network Bases of Epileptic Seizures. Annu. Rev. Physiol. 63, 815-846. https://doi.org/10.1146/annurev.physiol.63.1.815

Michalareas, G., Vezoli, J., van Pelt, S., Schoffelen, J.-M., Kennedy, H., Fries, P., 2016. AlphaBeta and Gamma Rhythms Subserve Feedback and Feedforward Influences among 
Human Visual Cortical Areas. Neuron 89, 384-397. https://doi.org/10.1016/j.neuron.2015.12.018

Minkwitz, J., Trenner, M.U., Sander, C., Olbrich, S., Sheldrick, A.J., Schonknecht, P., Hegerl, U., Himmerich, H., 2011. Prestimulus vigilance predicts response speed in an easy visual discrimination task. Behav. Brain Funct. BBF 7, 31. https://doi.org/10.1186/17449081-7-31

Moreau, J.T., Simard-Tremblay, E., Albrecht, S., Rosenblatt, B., Baillet, S., Dudley, R.W.R., 2020. Overnight ictal magnetoencephalography. Neurol. Clin. Pract. https://doi.org/10.1212/CPJ.0000000000000937

Morillon, B., Baillet, S., 2017. Motor origin of temporal predictions in auditory attention. Proc. Natl. Acad. Sci. 114, E8913-E8921. https://doi.org/10.1073/pnas.1705373114

Mostame, P., Sadaghiani, S., 2021. Oscillation-Based Connectivity Architecture Is Dominated by an Intrinsic Spatial Organization, Not Cognitive State or Frequency. J. Neurosci. 41, 179-192. https://doi.org/10.1523/JNEUROSCI.2155-20.2020

Mostame, P., Sadaghiani, S., 2020. Phase- and amplitude-coupling are tied by an intrinsic spatial organization but show divergent stimulus-related changes. Neurolmage 219, 117051. https://doi.org/10.1016/j.neuroimage.2020.117051

Murakami, S., Okada, Y., 2006. Contributions of principal neocortical neurons to magnetoencephalography and electroencephalography signals. J. Physiol. 575, 925936. https://doi.org/10.1113/jphysiol.2006.105379

Nentwich, M., Ai, L., Madsen, J., Telesford, Q.K., Haufe, S., Milham, M.P., Parra, L.C., 2020. Functional connectivity of EEG is subject-specific, associated with phenotype, and different from fMRI. Neurolmage 218, 117001. https://doi.org/10.1016/j.neuroimage.2020.117001

Nir, Y., Mukamel, R., Dinstein, I., Privman, E., Harel, M., Fisch, L., Gelbard-Sagiv, H., Kipervasser, S., Andelman, F., Neufeld, M.Y., Kramer, U., Arieli, A., Fried, I., Malach, R., 2008. Interhemispheric correlations of slow spontaneous neuronal fluctuations revealed in human sensory cortex. Nat. Neurosci. 11, 1100-1108. https://doi.org/10.1038/nn.2177

Niso, G., Bruña, R., Pereda, E., Gutiérrez, R., Bajo, R., Maestú, F., del-Pozo, F., 2013. HERMES: Towards an Integrated Toolbox to Characterize Functional and Effective Brain Connectivity. Neuroinformatics 11, 405-434. https://doi.org/10.1007/s12021-0139186-1

Nolte, G., Bai, O., Wheaton, L., Mari, Z., Vorbach, S., Hallett, M., 2004. Identifying true brain interaction from EEG data using the imaginary part of coherency. Clin. Neurophysiol. 115, 2292-2307. https://doi.org/10.1016/j.clinph.2004.04.029

Nunez, P.L., Srinivasan, R., 2006. Electric Fields of the Brain. Oxford University Press.

O'Neill, G.C., Bauer, M., Woolrich, M.W., Morris, P.G., Barnes, G.R., Brookes, M.J., 2015. Dynamic recruitment of resting state sub-networks. Neurolmage 115, 85-95. https://doi.org/10.1016/j.neuroimage.2015.04.030

O'Neill, G.C., Tewarie, P., Vidaurre, D., Liuzzi, L., Woolrich, M.W., Brookes, M.J., $2017 a$. Dynamics of large-scale electrophysiological networks: A technical review. Neurolmage. https://doi.org/10.1016/j.neuroimage.2017.10.003

O'Neill, G.C., Tewarie, P.K., Colclough, G.L., Gascoyne, L.E., Hunt, B.A.E., Morris, P.G., Woolrich, M.W., Brookes, M.J., 2017b. Measurement of dynamic task related functional networks using MEG. Neurolmage 146, 667-678. https://doi.org/10.1016/j.neuroimage.2016.08.061

Oostenveld, R., Fries, P., Maris, E., Schoffelen, J.-M., 2010. FieldTrip: Open Source Software for Advanced Analysis of MEG, EEG, and Invasive Electrophysiological Data. Comput. Intell. Neurosci. 2011. https://doi.org/10.1155/2011/156869 
O'Reilly, C., Elsabbagh, M., 2021. Intracranial recordings reveal ubiquitous in-phase and inantiphase functional connectivity between homotopic brain regions in humans. J. Neurosci. Res. 99, 887-897. https://doi.org/10.1002/jnr.24748

Palop, J.J., Chin, J., Mucke, L., 2006. A network dysfunction perspective on neurodegenerative diseases. Nature 443, 768-773. https://doi.org/10.1038/nature05289

Palva, J.M., Zhigalov, A., Hirvonen, J., Korhonen, O., Linkenkaer-Hansen, K., Palva, S., 2013. Neuronal long-range temporal correlations and avalanche dynamics are correlated with behavioral scaling laws. Proc. Natl. Acad. Sci. 110, 3585-3590. https://doi.org/10.1073/pnas.1216855110

Palva, S., Palva, J.M., 2012. Discovering oscillatory interaction networks with M/EEG: challenges and breakthroughs. Trends Cogn. Sci. 16, 219-230. https://doi.org/10.1016/j.tics.2012.02.004

Podvalny, E., Flounders, M.W., King, L.E., Holroyd, T., He, B.J., 2019. A dual role of prestimulus spontaneous neural activity in visual object recognition. Nat. Commun. 10, 3910. https://doi.org/10.1038/s41467-019-11877-4

Raichle, M.E., 2009. A Paradigm Shift in Functional Brain Imaging. J Neurosci 29, 12729_ 12734. https://doi.org/10.1523/JNEUROSCI.4366-09.2009

Rajagovindan, R., Ding, M., 2011. From prestimulus alpha oscillation to visual-evoked response: an inverted-U function and its attentional modulation. J. Cogn. Neurosci. 23, 1379-1394. https://doi.org/10.1162/jocn.2010.21478

Rasch, M., Logothetis, N.K., Kreiman, G., 2009. From Neurons to Circuits: Linear Estimation of Local Field Potentials. J. Neurosci. 29, 13785-13796. https://doi.org/10.1523/JNEUROSCI.2390-09.2009

Rassi, E., Wutz, A., Müller-Voggel, N., Weisz, N., 2019. Prestimulus feedback connectivity biases the content of visual experiences. Proc. Natl. Acad. Sci. 116, 16056-16061. https://doi.org/10.1073/pnas.1817317116

Rimmele, J.M., Morillon, B., Poeppel, D., Arnal, L.H., 2018. Proactive Sensing of Periodic and Aperiodic Auditory Patterns. Trends Cogn. Sci., Special Issue: Time in the Brain 22, 870-882. https://doi.org/10.1016/j.tics.2018.08.003

Romei, V., Gross, J., Thut, G., 2010. On the Role of Prestimulus Alpha Rhythms over OccipitoParietal Areas in Visual Input Regulation: Correlation or Causation? J Neurosci 30, 8692-8697. https://doi.org/10.1523/JNEUROSCI.0160-10.2010

Ruzzoli, M., Torralba, M., Morís Fernández, L., Soto-Faraco, S., 2019. The relevance of alpha phase in human perception. Cortex 120, 249-268. https://doi.org/10.1016/j.cortex.2019.05.012

Sadaghiani, S., Dombert, P.L., Løvstad, M., Funderud, I., Meling, T.R., Endestad, T., Knight, R.T., Solbakk, A.-K., D’Esposito, M., 2019. Lesions to the Fronto-Parietal Network Impact Alpha-Band Phase Synchrony and Cognitive Control. Cereb. Cortex 29, 41434153. https://doi.org/10.1093/cercor/bhy296

Sadaghiani, S., Hesselmann, G., Friston, K.J., Kleinschmidt, A., 2010. The relation of ongoing brain activity, evoked neural responses, and cognition. Front. Syst. Neurosci. 4. https://doi.org/10.3389/fnsys.2010.00020

Sadaghiani, S., Kleinschmidt, A., 2016. Brain Networks and $\alpha$-Oscillations: Structural and Functional Foundations of Cognitive Control. Trends Cogn. Sci. 20, 805-817. https://doi.org/10.1016/j.tics.2016.09.004

Sadaghiani, S., Kleinschmidt, A., 2013. Functional interactions between intrinsic brain activity and behavior. Neurolmage 80, 379-386. https://doi.org/10.1016/j.neuroimage.2013.04.100

Sadaghiani, S., Wirsich, J., 2019. Intrinsic connectome organization across temporal scales: New insights from cross-modal approaches. Netw. Neurosci. 4, 1-29. https://doi.org/10.1162/netn_a_00114 
Sareen, E., Zahar, S., Ville, D.V.D., Gupta, A., Griffa, A., Amico, E., 2021. Exploring MEG brain fingerprints: evaluation, pitfalls and interpretations. bioRxiv 2021.02.15.431253. https://doi.org/10.1101/2021.02.15.431253

Schnitzler, A., Gross, J., 2005. Functional Connectivity Analysis in Magnetoencephalography, in: International Review of Neurobiology, Magnetoencephalography. Academic Press, pp. 173-195. https://doi.org/10.1016/S0074-7742(05)68007-5

Schoffelen, J.-M., Gross, J., 2009. Source connectivity analysis with MEG and EEG. Hum. Brain Mapp. 30, 1857-1865. https://doi.org/10.1002/hbm.20745

Schoffelen, J.-M., Oostenveld, R., Fries, P., 2005. Neuronal Coherence as a Mechanism of Effective Corticospinal Interaction. Science 308, 111-113. https://doi.org/10.1126/science.1107027

Schölvinck, M.L., Maier, A., Ye, F.Q., Duyn, J.H., Leopold, D.A., 2010. Neural basis of global resting-state fMRI activity. Proc. Natl. Acad. Sci. 107, 10238-10243. https://doi.org/10.1073/pnas.0913110107

Schroeder, C.E., Wilson, D.A., Radman, T., Scharfman, H., Lakatos, P., 2010. Dynamics of Active Sensing and perceptual selection. Curr. Opin. Neurobiol., Cognitive neuroscience 20, 172-176. https://doi.org/10.1016/j.conb.2010.02.010

Segneri, M., Bi, H., Olmi, S., Torcini, A., 2020. Theta-Nested Gamma Oscillations in Next Generation Neural Mass Models. Front. Comput. Neurosci. 14, 47. https://doi.org/10.3389/fncom.2020.00047

Siegel, M., Donner, T.H., Engel, A.K., 2012. Spectral fingerprints of large-scale neuronal interactions. Nat Rev Neurosci 13, 121-134. https://doi.org/10.1038/nrn3137

Sporns, O., 2010. Networks of the Brain | The MIT Press. The MIT Pres.

Stam, C.J., Nolte, G., Daffertshofer, A., 2007. Phase lag index: Assessment of functional connectivity from multi channel EEG and MEG with diminished bias from common sources. Hum. Brain Mapp. 28, 1178-1193. https://doi.org/10.1002/hbm.20346

Steriade, M., Gloor, P., Llinás, R.R., Lopes da Silva, F.H., Mesulam, M.-M., 1990. Basic mechanisms of cerebral rhythmic activities. Electroencephalogr. Clin. Neurophysiol. 76, 481-508. https://doi.org/10.1016/0013-4694(90)90001-Z

Tewarie, P., Bright, M.G., Hillebrand, A., Robson, S.E., Gascoyne, L.E., Morris, P.G., Meier, J., Van Mieghem, P., Brookes, M.J., 2016. Predicting haemodynamic networks using electrophysiology: The role of non-linear and cross-frequency interactions. Neurolmage 130, 273-292. https://doi.org/10.1016/j.neuroimage.2016.01.053

Tewarie, P., Hunt, B.A.E., O’Neill, G.C., Byrne, A., Aquino, K., Bauer, M., Mullinger, K.J., Coombes, S., Brookes, M.J., 2019. Relationships Between Neuronal Oscillatory Amplitude and Dynamic Functional Connectivity. Cereb. Cortex 29, 2668-2681. https://doi.org/10.1093/cercor/bhy136

Thiery, T., Saive, A.-L., Combrisson, E., Dehgan, A., Bastin, J., Kahane, P., Berthoz, A., Lachaux, J.-P., Jerbi, K., 2020. Decoding the neural dynamics of free choice in humans. PLOS Biol. 18, e3000864. https://doi.org/10.1371/journal.pbio.3000864

Trujillo, C.A., Gao, R., Negraes, P.D., Gu, J., Buchanan, J., Preissl, S., Wang, A., Wu, W., Haddad, G.G., Chaim, I.A., Domissy, A., Vandenberghe, M., Devor, A., Yeo, G.W., Voytek, B., Muotri, A.R., 2019. Complex Oscillatory Waves Emerging from Cortical Organoids Model Early Human Brain Network Development. Cell Stem Cell 25, 558569.e7. https://doi.org/10.1016/j.stem.2019.08.002

Uhlhaas, P.J., Singer, W., 2010. Abnormal neural oscillations and synchrony in schizophrenia. Nat Rev Neurosci 11, 100-113. https://doi.org/10.1038/nrn2774

van Diepen, R.M., Cohen, M.X., Denys, D., Mazaheri, A., 2015. Attention and Temporal Expectations Modulate Power, Not Phase, of Ongoing Alpha Oscillations. J. Cogn. Neurosci. 27, 1573-1586. https://doi.org/10.1162/jocn_a_00803 
Vicente, R., Gollo, L.L., Mirasso, C.R., Fischer, I., Pipa, G., 2008. Dynamical relaying can yield zero time lag neuronal synchrony despite long conduction delays. Proc. Natl. Acad. Sci. 105, 17157-17162. https://doi.org/10.1073/pnas.0809353105

Vidaurre, D., Hunt, L.T., Quinn, A.J., Hunt, B.A.E., Brookes, M.J., Nobre, A.C., Woolrich, M.W., 2018. Spontaneous cortical activity transiently organises into frequency specific phasecoupling networks. Nat. Commun. 9, 2987. https://doi.org/10.1038/s41467-018-05316-z

Weaver, K.E., Wander, J.D., Ko, A.L., Casimo, K., Grabowski, T.J., Ojemann, J.G., Darvas, F., 2016. Directional patterns of cross frequency phase and amplitude coupling within the resting state mimic patterns of fMRI functional connectivity. Neurolmage 128, 238-251. https://doi.org/10.1016/j.neuroimage.2015.12.043

Weisz, N., Wühle, A., Monittola, G., Demarchi, G., Frey, J., Popov, T., Braun, C., 2014. Prestimulus oscillatory power and connectivity patterns predispose conscious somatosensory perception. Proc. Natl. Acad. Sci. 111, E417-E425. https://doi.org/10.1073/pnas.1317267111

Wirsich, J., Giraud, A.-L., Sadaghiani, S., 2020. Concurrent EEG- and fMRI-derived functional connectomes exhibit linked dynamics. Neurolmage 219, 116998. https://doi.org/10.1016/j.neuroimage.2020.116998

Wirsich, J., Jorge, J., lannotti, G.R., Shamshiri, E.A., Grouiller, F., Abreu, R., Lazeyras, F., Giraud, A.-L., Gruetter, R., Sadaghiani, S., Vulliémoz, S., 2021. The relationship between EEG and fMRI connectomes is reproducible across simultaneous EEG-fMRI studies from $1.5 \mathrm{~T}$ to $7 \mathrm{~T}$. Neurolmage $231,117864$. https://doi.org/10.1016/j.neuroimage.2021.117864

Wirsich, J., Ridley, B., Besson, P., Jirsa, V., Bénar, C., Ranjeva, J.-P., Guye, M., 2017. Complementary contributions of concurrent EEG and fMRI connectivity for predicting structural connectivity. Neurolmage 161, 251-260. https://doi.org/10.1016/j.neuroimage.2017.08.055

Woolrich, M.W., Baker, A., Luckhoo, H., Mohseni, H., Barnes, G., Brookes, M., Rezek, I., 2013. Dynamic state allocation for MEG source reconstruction. Neurolmage 77, 77-92. https://doi.org/10.1016/j.neuroimage.2013.03.036

Yeung, R.W., 2002. A First Course in Information Theory, Information Technology: Transmission, Processing and Storage. Springer US. https://doi.org/10.1007/978-14419-8608-5

Yuen, N.H., Osachoff, N., Chen, J.J., 2019. Intrinsic Frequencies of the Resting-State fMRI Signal: The Frequency Dependence of Functional Connectivity and the Effect of Mode Mixing. Front. Neurosci. 13. https://doi.org/10.3389/fnins.2019.00900

Zhigalov, A., Herring, J.D., Herpers, J., Bergmann, T.O., Jensen, O., 2019. Probing cortical excitability using rapid frequency tagging. Neurolmage 195, 59-66. https://doi.org/10.1016/j.neuroimage.2019.03.056 